\title{
Emerging efficient charge-transport landscape based on short-range order in conjugated polymers
}

Suhao Wang

The self-archived postprint version of this journal article is available at Linköping University Institutional Repository (DiVA):

http://urn.kb.se/resolve?urn=urn:nbn:se:liu:diva-156696

N.B.: When citing this work, cite the original publication.

Wang, S., (2019), Emerging efficient charge-transport landscape based on short-range order in conjugated polymers, Synthetic metals, 251, 104-119. https://doi.org/10.1016/j.synthmet.2019.03.021

Original publication available at:

https://doi.org/10.1016/j.synthmet.2019.03.021

Copyright: Elsevier

http://www.elsevier.com/

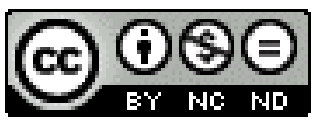




\title{
Emerging efficient charge-transport landscape based on short- range order in conjugated polymers
}

Suhao Wang*

Laboratory of Organic Electronics, Department of Science and Technology, Linköping University, SE-60174 Norrköping, Sweden

${ }^{*}$ Corresponding author.

E-mail address: suhao.wang@liu.se

\section{Highlights}

- Milestones are focus-reviewed by highlighting most remarkable conjugated polymers.

- Emerging charge-transport models based on short-range order are summarized.

- Recent experimental progress based on short-range order is highlighted.

\begin{abstract}
:
The booming of classic semicrystalline polymers has led to the assumption and thus design guidelines that long-range order is a prerequisite to endow conjugated polymers with high charge carrier mobility. Consequently, tremendous research effort has been devoted to increasing the crystallinity of conjugated polymers, as a principal strategy to improve the solidstate long-range charge-transport properties. Indeed, noticeable progress in the polymer performance has been witnessed. However, only a limited level of crystallinity can be achieved due to the inherently disordered nature of the polymer chains, resulting in the bottlenecks of the charge carrier mobility of conjugated polymers. Encouragingly, the recent reports of substantially disordered, high-performance conjugated polymers have opened a new route for achieving efficient charge transport, and lead to new waves of progress in the field of organic electronics. The universal observation of short-range order (in the form of aggregation) in the emerging class of poorly ordered conjugated polymers seems to suggest that local order is sufficient for efficient charge transport, and that extended long-range crystallinity is not essential. This review discusses the molecular origin of the high mobilities observed in the state-of-the-art low-crystalline conjugated polymers, especially highlighting the crucial role of short-range order.
\end{abstract}

Key words:

Donor-acceptor polymers; Short-range order; Aggregation; Seemingly disordered; High mobility; Organic field effect transistors 


\section{Introduction:}

Over the past three decades, organic field-effect transistors (OFETs) based on conjugated semiconducting polymers have attracted significant scientific and industrial attentions, as they show great potential in enabling large-area fabrication of flexible electronic devices via easy solution processing[1-5]. Charge carrier mobility, the key figure of merit for conjugated polymers, has been boosted by $>4$ orders of magnitude, mainly thanks to continuous efforts in molecular design[6-9], as well as the advances of solution processing[10-12]. The state-of-theart mobilities are now $>10 \mathrm{~cm}^{2} \mathrm{~V}^{-1} \mathrm{~s}^{-1}[13-21]$, surpassing that of benchmark thin-film amorphous silicon $\left(0.5-1 \mathrm{~cm}^{2} \mathrm{~V}^{-1} \mathrm{~s}^{-1}\right)$ (Fig. 1). Alongside experiencing the breakthroughs of polymer performance, organic electronics community also gained better understanding of molecular designing rules[22-25] and witnessed the dramatic evolution of charge-transport models[25-27]. The goal of this review is to highlight the recent achievements in the materials breakthroughs and discuss the molecular origin of emerging efficient charge-transport landscape. Particularly, milestones that lead to the state-of-the-arts mobilities, evolution in charge-transport models, as well as recent experimental progress are presented by highlighting several most remarkable conjugated polymers.

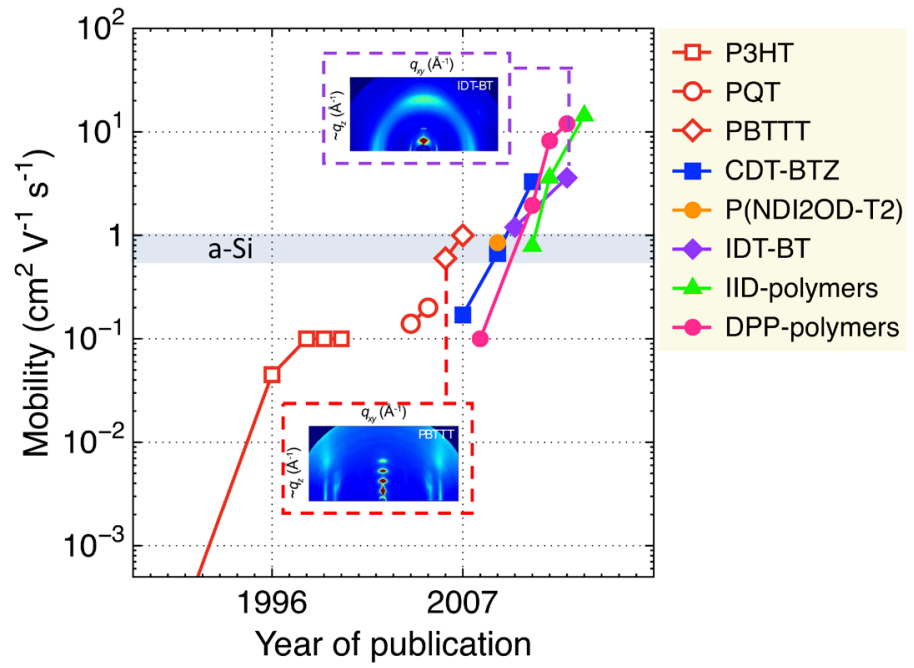

Fig. 1. Evolution of charge carrier mobilities (those $<10^{-3} \mathrm{~cm}^{2} \mathrm{~V}^{-1} \mathrm{~s}^{-1}$ are not shown) for the semicrystalline polymers (P3HT, PQT, PBTTT, shown in red, open) and emerging poorly ordered high performing polymers (shown in other colors, filled), plotted as a function of the year in the past 3 decades. The year 1996, and 2007 represent the start of the two milestones. The mobilities in this figure are obtained from reports where conjugated polymers were processed with common solvents and casting techniques (without chain alignment). (Insets: 2D-GIXD patterns of PBTTT (bottom), and IDT-BT (top)) 2D-GIXD patterns: reproduced with permission from Ref.[27]. Copyright (C) 2013, Macmillan Publishers Ltd.

Initially, the development of conjugated polymers was based on the investigation of amorphous thin-films of polymers, such as regiorandom poly(3-heylthiophene) (r-Ra P3HT) $[28,29]$ and polyparaphenylenevinylenes (PPV) derivatives[30, 31], yielding relatively low mobility $\left(<10^{-}\right.$ $\left.{ }^{3} \mathrm{~cm}^{2} \mathrm{~V}^{-1} \mathrm{~s}^{-1}\right)$. The booming stage of conjugated polymer development was marked by the most successful demonstration of regioregular (r-Re) P3HT (Fig. 2A), a high-performance, solutionprocessable polymer yielding a mobility up to $\approx 0.1 \mathrm{~cm}^{2} \mathrm{~V}^{-1} \mathrm{~s}^{-1}[32-35]$. The striking crystallinity of this benchmark polymer has led to the assumption that long-range order is a prerequisite for conjugated polymers to achieve high mobility. Hereby, adjacent polymer chains, being packed face-to-face (commonly comprehended as $\pi-\pi$ stacking) in a lamellar 
fashion, can either be face-on (Fig. 2D, with the backbone plane being parallel to the substrate) or edge-on (Fig. 2E, with the backbone plane being perpendicular to the substrate). More specifically, a two-dimensional (2D) charge-transport model with edge-on lamellar microstructure (Fig. 2F) has been widely assumed to be essential for efficient charge transport, that charge transport takes place both along the conjugated backbone direction (axis a, Fig. 2F), and along the in-plane $\pi-\pi$ stacking direction (axis b, Fig. 2F). In the view of the anisotropic charge transport in conjugated polymers (fastest along axis a, moderate along axis $b$, and slowest along the chain to chain direction(axis c), Fig. 2F) [3, 10, 36, 37], 2D charge-transport model is most favorable for charge transport since in this scenario the difficult interchain hopping steps between conjugated units separated by the insulating side chains can be minimized. Indeed, a much inferior mobility of $2 \times 10^{-4} \mathrm{~cm}^{2} \mathrm{~V}^{-1} \mathrm{~s}^{-1}$ was measured when $\mathrm{r}$-Re P3HT lays face-on the substrate, despite the pronounced in-plane crystallinity along the (100)axis indicated by XRD[33]. Later, the report of analogous polymer poly(3,3'-dialkylquaterthiophene) (PQT) [38, 39] (Fig. 2B), with similar edge-on lamellar structures and slightly better mobility of $\approx 0.2 \mathrm{~cm}^{2} \mathrm{~V}^{-1} \mathrm{~s}^{-1}[39]$, has made the $2 \mathrm{D}$ charge-transport model more convincing.
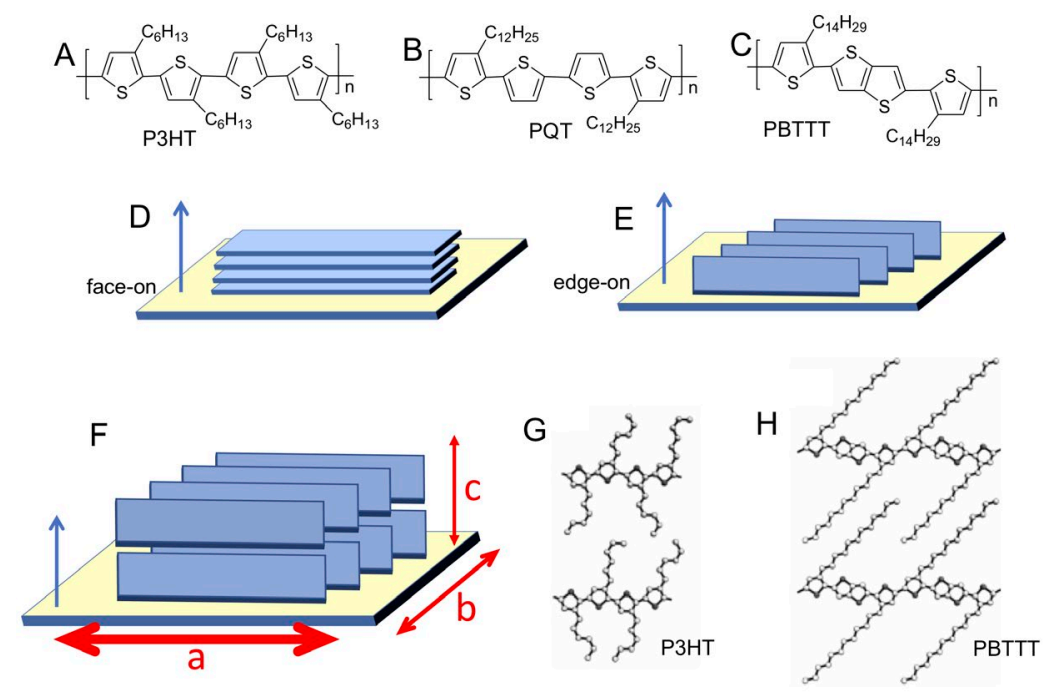

Fig. 2. Chemical structures of classic semicrystalline conjugated polymers: (A) P3HT, (B) PQT and (C) PBTTT. (D) face-on and (E) edge-on stacking texture of conjugated polymers. (polymer side-chains are omitted from the schematic model, and the blue arrows denote the film surface normal direction.) (F) 2D-charge transport model with edge-on lamellar microstructure. Illustration of side-chains packing of (G) P3HT, and (H) PBTTT. (G) and (H):Adapted from Ref.[40] with permission. Copyright (C) 2007, American Chemical Society.

Consequently, for over a decade the research strategy to improve the solid-state chargetransport properties has primarily been increasing the long-range crystallinity of conjugated polymers. Admittedly, under this guideline the charge carrier mobility has increased noticeably by almost 1 order of magnitude, with more highly crystalline poly(2,5-bis(thiophen-2yl)thieno-[3,2-b]thiophene) (PBTTT) as a most outstanding validation (Fig. 2C), yielding higher mobility up to $\approx 1 \mathrm{~cm}^{2} \mathrm{~V}^{-1} \mathrm{~s}^{-1}[41,42]$. Interestingly, PBTTT exhibits an in-plane liquid crystalline texture and its more highly crystalline structures are ascribed to the lower density of side chains leading to greater side-chain interdigitation[40] (Fig. 2G, H). A typical 2D grazing incidence X-ray diffraction (2D-GIXD) pattern of PBTTT is featured by several welldefined diffraction peaks (Fig.1(inset, bottom)). However, further attempts to improve the mobility by increasing crystallinity has been hampered by the inherently disordered nature of the polymers. It is reported that only a limited level of crystallinity can be achieved for highly 
crystalline polymers[43-45]. The random chain packing in the amorphous regions were assumed to block the connection of conjugated backbone, thus hindering charge carrier delocalization and limiting the charge carrier mobility.

In general, the most acknowledged paradigms established by the early work on classic semicrystalline conjugated polymers (P3HT, PQT and PBTTT) are often correlated to homopolymer, high regioregularity, high crystallinity, long-range order, edge-on lamellar microstructure, 2-D transport[46]. Accordingly, these polymers are quite sensitive to disorder so that their mobilities can be severely affected by poor chain-packing and lack of long-range order. A prominent example is that the highly ordered r-Re P3HT exhibits significantly higher mobility than its amorphous counterpart ( $\mathrm{r}-\mathrm{Ra}$ P3HT) by $>4$ orders of magnitude[28, 32]. Moreover, the essential role of molecular weight has been recognized through that increasing molecular weight of r-Re P3HT by ca.1 order of magnitude resulted in a pronounced increase in the mobility by ca. 4 orders of magnitude[47]. Besides, a well-controlled polydispersity (PDI) is highly desirable for achieving efficient charge transport, since even small amounts of low molecular weight material can have detrimental effects on mobilities, as indicated by molecular mixing of high and low molecular weight P3HT chains[48]. Together, these sensitive features probably can explain why these polymers suffer from lab-to-lab and batch-to-batch variability, as shown by the survey of over ten years of literature data[27]. In addition, the activation energies measured in these highly ordered polymers ( $r$-Re P3HT, PBTTT) are usually in the range of $\approx 50-70 \mathrm{meV}[47,49]$. It is worth recalling that in OFETs, activation energies derived from temperature-dependent saturation mobilities are widely used to indicate the energy between occupied traps and mobile states. Usually the larger the activation energies, the greater the traps.

Recently, however, the emergence of substantially disordered polymers-that can rival or even outperform the best classic ordered polymers-has puzzled both experimentalists and theoreticians, motivated further efforts to reassess the structural-functional properties paradigm, and lead to a new wave of progress in the field of organic electronics. Compared to aforementioned classic semicrystalline polymers, the emerging host of conjugated polymers have more complex backbone structures, consisting of alternating electron-rich donor and electron-deficient acceptor units (namely D-A copolymers)[50], which initially have been merely investigated in organic photovoltaic (OPV) devices, thanks to their attractive low band gap [51, 52]. Moreover, the new class of polymers often exhibit lower crystallinity, as featured by larger breadth of diffraction peaks (Fig.1(inset, top)). Besides, the large and planar conjugated backbone structure usually helps promote a closer $\pi$ stacking distance, facilitating intermolecular charge transfer. In recent years, a wide range of D-A copolymers appeared as promising candidates for OEFTs application. Pioneering works are based on copolymer of cyclopentadithiophene and benzothiadiazole (CDT-BTZ)[53], copolymer of naphthalenediimide and bithiophene (P(NDI2OD-T2))[54], copolymer of indacenodithiophene and benzothiadiazole (IDT-BT)[55], and a bunch of copolymers based on diketopyrrolopyrrole (DPP)[56], isoindigo (IID)[57], etc.

While there are many other high-performance conjugated polymers that have been published in recent years, this review does not aim at comprehensively covering the literature. Rather, it provides a picture clarifying the origin of the puzzling high mobility observed in the new host of poorly ordered materials, through reviewing some most representative exemplary polymers. Therefore, the author regrets the omission of other excellent literature in this review. Before moving onto the following chapters, it is also important to note that this review is not to undervalue the importance of long-range crystallinity, but instead to put a particular emphasis 
on the crucial role of short-range order: that high mobility can be achieved in poorly ordered conjugated polymers comprising short-range ordered interconnected aggregates, representing a new pathway to realize efficient charge transport. Especially, the crucial role of short-range order seems to be the dominant one that is playing in many of the recent high-mobility conjugated polymers.

In chapter 2, a brief review of emerging low-crystalline, high-performance conjugated polymers is presented. Then, proposals for emerging charge-transport models are summarized in chapter 3. After that, some recent experimental progress, promoted by short-range order, is highlighted in chapter 4. Finally, in chapter 5, this review is concluded with an outlook for future challenges and opportunities.

\section{Emerging weakly ordered, high-performance conjugated polymers}

\subsection{Benzothiadiazole-based copolymers}

\subsubsection{Copolymer of cyclopentadithiophene and benzothiadiazole}

The first demonstration of D-A copolymer in OFETs application is the seemingly amorphous copolymer CDT-BTZ (P1, Fig. 3)[53]. Substantial disorder was indicated by the lack of XRD scattering characteristics[53] and later confirmed by the quasi-featureless GIWAXS patterns of spin coated P1 film[58]. Rather counterintuitively, such a poorly ordered CDT-BTZ film exhibited an impressive mobility of $0.17 \mathrm{~cm}^{2} \mathrm{~V}^{-1} \mathrm{~s}^{-1}$, even outperforming best ordered, highly crystalline benchmark polymer P3HT. It is noteworthy that, for device fabrication, the polymer was casually processed with conventional casting method (drop casting) from common solvent [53]. The pronounced mobility was mainly ascribed to the small $\pi-\pi$ stacking distance of $3.7 \AA$, as deduced from 2D wide-angle X-ray scattering (2D-WAXS) measurement. Such a close packing is most likely promoted by the highly planar backbone confirmation of P1, which minimizes the structural disorder. Hereby, small face-to-face distance allows better molecular orbital overlap, which is nowadays widely used to enhance the charge carrier mobility[59]. Although the microstructural origin of the observed high mobility was not fully articulated at the time of the report, the emergence of $\mathbf{P 1}$ did suggest a new route to achieve efficient chargetransport, that it is attainable when polymer chains do not possess long-range intermolecular order but are locally aggregated.
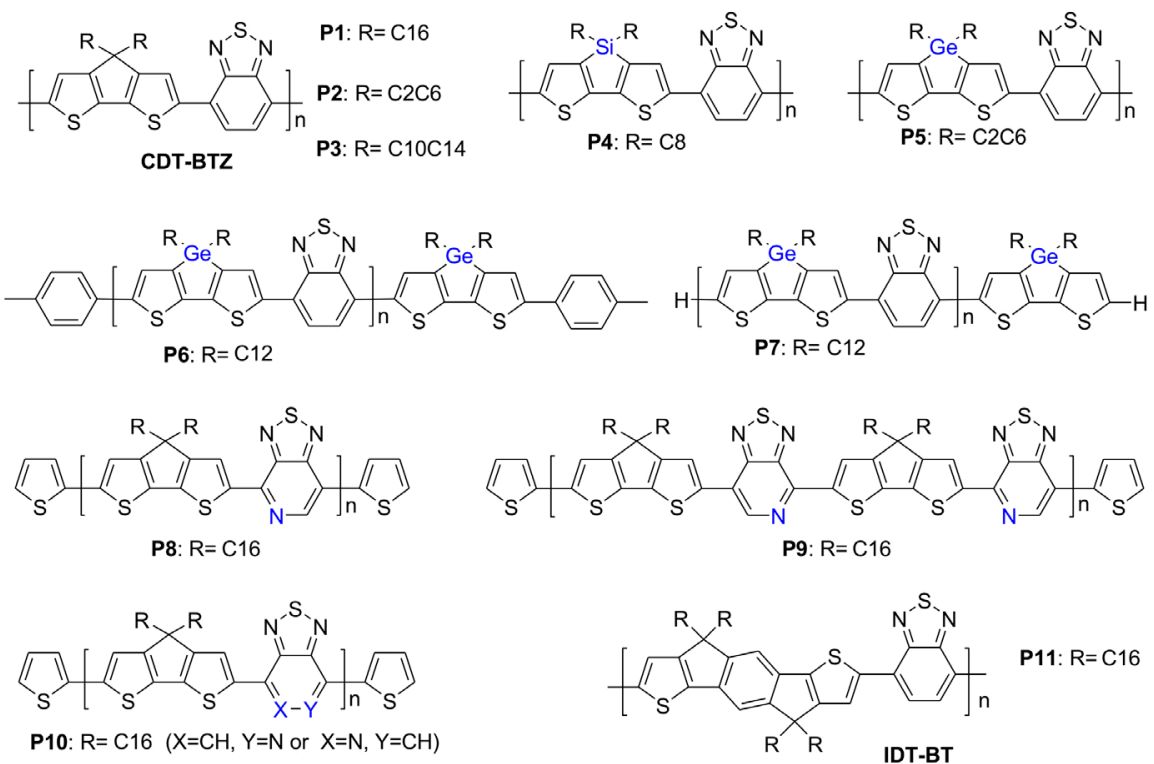

Fig. 3. Chemical structures of BT-based copolymers. Acronyms for side chains: C8(1-octyl), C12(1-dodecyl), C16(1-hexadecyl), C2C6(2-ethyl-1-hexyl), C10C14(2-decyl-1-tetradecyl). 

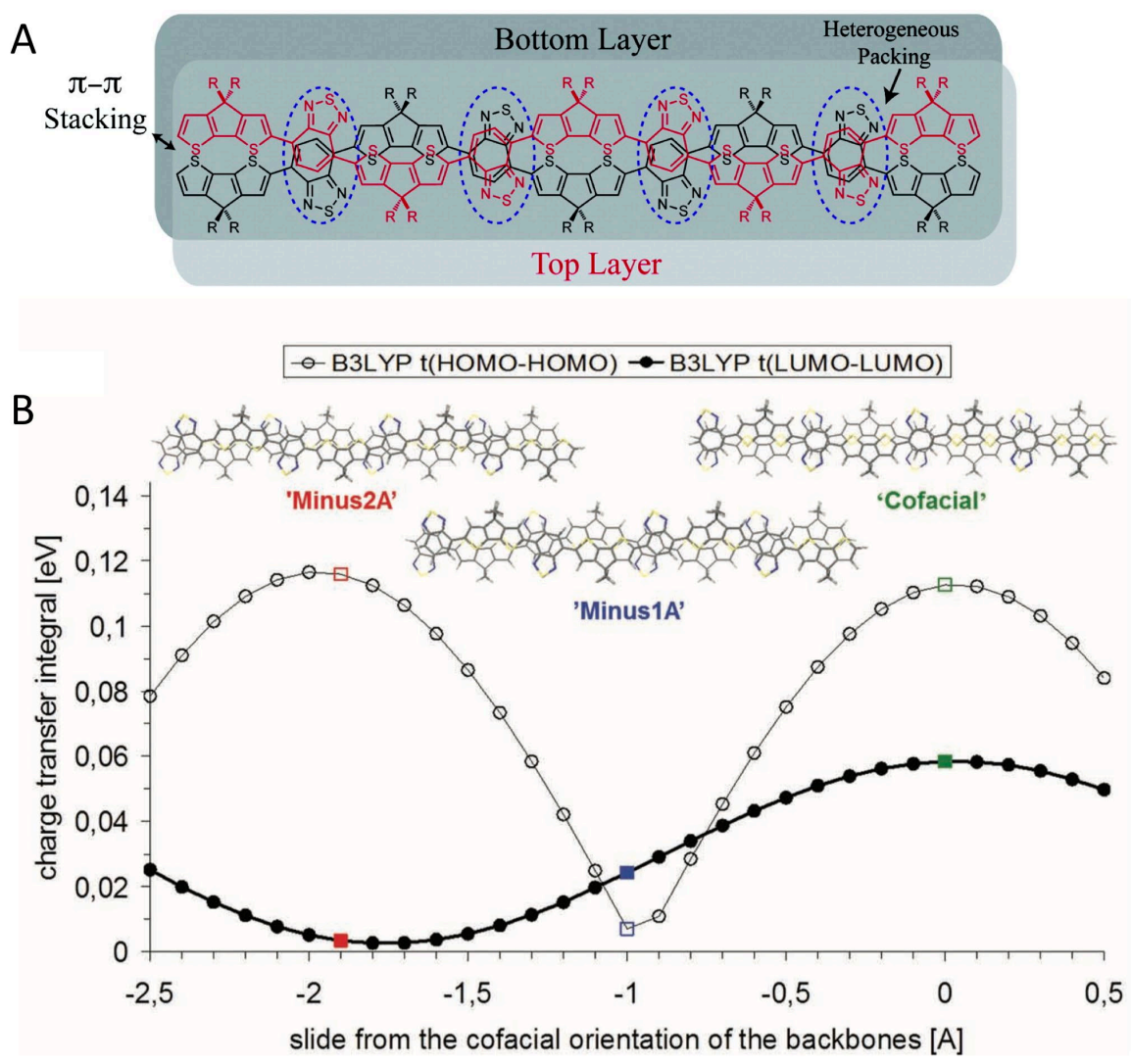

Fig. 4. (A) Schematic drawing illustrating the local packing of donor-acceptor groups in two neighboring P1 copolymer chains (here denoted as "layers"). The dashed circles (blue) mark the regions where the acceptors groups are heterogeneously packed on top of one another. Reprinted with permission from Ref.[60] Copyright (C) 2011 American Chemical Society. (B) Charge transfer integral (absolute value, in $\mathrm{eV}$ ) between P1 tetramers, as calculated at the DFT/B3LYP level for different sliding distances of the conjugated backbones ( 0 corresponds to the cofacial structure). Reprinted with permission from Ref.[61] Copyright (C) 2013 Wiley.

Rational design of the polymer structure, e.g. increasing molecular weight and reducing the PDI of P1, has led to a significant enhancement in the mobility, reaching a remarkable value of $3.3 \mathrm{~cm}^{2} \mathrm{~V}^{-1} \mathrm{~s}^{-1}[60]$. For the first time 2-D nuclear magnetic resonance (2D-NMR) was employed to deduce the donor-acceptor interactions between neighboring polymer chains, indicating an acceptor-to-acceptor stacking motif (Fig. 4A), rather than initially hypothesized donor-to-acceptor stacking. By bridging together GIXD and 2D-NMR data in conjugation with density functional theory (DFT) molecular modelling, a refined predominantly donor-to-donor and acceptor-to-acceptor motif with a small longitudinal shift ( $2 \AA)$ was identified, which favorably happened to exhibit the largest transfer integrals (Fig. 4B)[61]. Such an arrangement was to a large extent triggered by van der Waals interactions between the long, linear, alkyl chains, rather than electrostatic donor-acceptor interactions[61]. Importantly, it was shown how significantly the subtle longitudinal shifts of the polymer backbones could affect the intermolecular electron coupling and thus the experimentally measured mobilities, emphasizing that the mobility of conjugated polymers is dictated by their structure on the $\AA$ scale. In other words, closer packing itself is not sufficient to ensure efficient intermolecular charge transfer, thus reminding chemists to take the uncertain backbone shifts into consideration and design molecules that are more resilient to these shifts. 
In addition, both side-chain engineering and backbone heteroatoms replacement allow tuning the intermolecular packing of CDT-BTZ and the corresponding device performance. For instance, using branched alkyl chains (P2, P3) instead of linear side chains (P1) increased the $\pi-\pi$ stacking distance and endowed the polymer with ambipolarity[62, 63], whereas silolecontaining copolymer (P4) [64] and germole-containing copolymer (P5) [65] exhibited closer $\pi-\pi$ stacking distance compared to the carbon analogue. Furthermore, end capping was also proven to play an important role in dominating the molecular order and microstructure (P6), leading to mobilities up to ca. 1 order of magnitude higher in comparison to that of the nonend-capped analogue (P7) [66]. Interestingly, regioregularity has a striking effect on the mobilities of pyridal-containing analogous polymers, yielding a rather noticeable difference of ca. 2 orders of magnitude between r-Re $(\mathbf{P 8}, \mathbf{P 9})$ and r-Ra (P10) structures[67]. Due to the limited space of this review, broader overview can be found in a recent review which has summarized a decade of research on CDT-BTZ[68].

\subsubsection{Copolymer of indacenodithiophene and benzothiadiazole}

Using more planar and larger IDT as donor unit yielded another weakly ordered, high-mobility, BT-based copolymer IDT-BT (P11)[55]. A small torsional angle of just $\approx 7^{\circ}$ has been predicated by DFT calculations, suggesting a quasi-planer conformation of P11 $[55,69]$. The diffuse ring observed from the GIXS image indicates that the P11 has a lack of pronounced long-range crystallinity. Nevertheless, this polymer gives an outstanding mobility of $1.2 \mathrm{~cm}^{2}$ $\mathrm{V}^{-1} \mathrm{~s}^{-1}$ [55], outperforming the best-ordered polymer PBTTT. Interestingly, the P11 backbones were found to align preferably face-on the substrate, which appears to contradict the conventional edge-on 2-D transport paradigms established for achieving high mobility. Although the insulating side chains laying in plane will surely form barriers for charge transport, the fastest charge-transport direction along the backbone is still predominantly in-plane. Moreover, the close $\pi-\pi$ stacking in the direction perpendicular to the interface will enable polymer layers, other than the first monolayer adjacent to the gate dielectric, to get involved in transport[25]. The backbone coplanarity of P11 was assumed to favor intramolecular charge delocalization. Later, a much higher mobility of $3.6 \mathrm{~cm}^{2} \mathrm{~V}^{-1} \mathrm{~s}^{-1}$ has been achieved as a result of increased molecular mass and smaller PDI[70]. Despite lack of long-range order, activation energy extracted from the Arrhenius plot was found to be merely $61 \mathrm{meV}$, comparable to that of the classic semicrystalline polymers[47, 49]. With the help of combined diffraction and polarizing spectroscopic techniques, for the first time a quasi-1D charge-transport model based on the study of P11 was proposed[70]. More details will be presented and discussed in section 3.1. Furthermore, through another study based on P11, the importance of backbone rigidity was evidenced and molecular-design guidelines for 'disorder-free' conjugated polymers were suggested[71], which will be focused in section 3.3.

\subsection{Copolymers of naphthalenediimide and bithiophene}

The most outstanding n-type electron-transporting copolymer is the high-performance P(NDI2OD-T2) (P12, Fig. 5). In contrast with the aforementioned planar BT-based copolymers, $\mathbf{P 1 2}$ is structurally distorted, with pronounced dihedral angles between the single conjugated units induced by steric crowding between the NDI2OD and the T2 moieties[72, 73]. In the bulk of the films the polymer backbones were found to lay preferentially face-on with respect to the substrate[74](Fig. 6A). However, distinct edge-on preferential orientation of the conjugated polymer backbone was revealed at the film surface[75, 76]. An exceptionally high electron mobility of $0.85 \mathrm{~cm}^{2} \mathrm{~V}^{-1} \mathrm{~s}^{-1}$ was achieved in top-gate transistors[77]. It was argued that charges were able to hop in the vertical directions via $\pi-\pi$ stacking, supplying an additional route for charge transport in contrast to lamellar packing as exhibited by P3HT[74]. Annealing the $\mathbf{P 1 2}$ film to its melting point, followed by slow cooling to ambient temperature, leads to a 
shift from predominantly face-on to mostly edge-on lamellar texture (Fig. 6B). Remarkably, a variety of X-ray diffraction analyses, including pole figure construction and Fourier transform peak shape deconvolution, allow for the quantification of the texture change(from $\sim 77 \%$ faceon crystallites to $94.5 \%$ edge-on), relative degree of crystallinity(2-fold increase in crystallinity), as well as lattice order changes (a $40 \%$ decrease in intracrystallite cumulative disorder)[78]. Correspondingly, a significant drop was observed in the electron-only diode current density through the film thickness upon melt annealing, while little change was detected in the in-plane transport of bottom gated thin film transistors. This was attributed to the fact that the texture is different between bottom surface structure and the interior structure or that charge transport in $\mathbf{P 1 2}$ is insensitive to the intracrystalline order and texture[78].
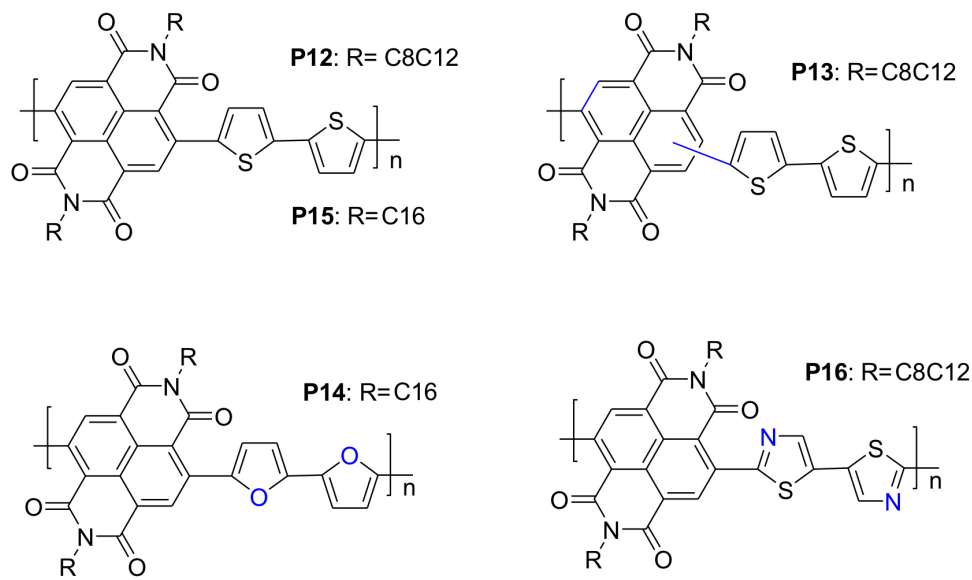

Fig. 5. Chemical structures of P(NDI2OD-T2) and analogous copolymers. Acronyms for side chains: C16(1-hexadecyl), C8C12(2-octyl-1-dodecyl).

A

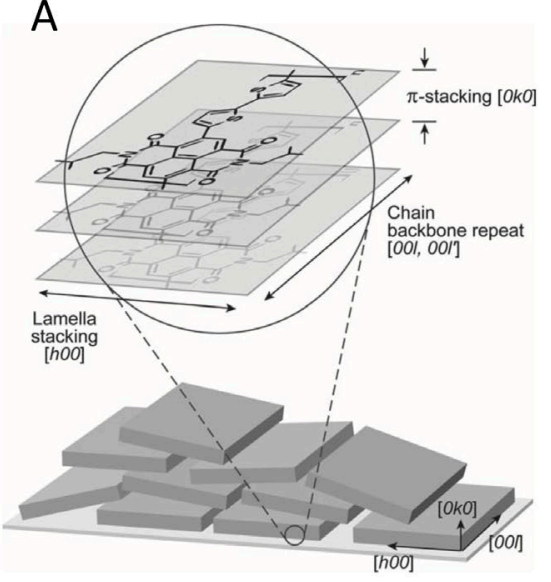

B

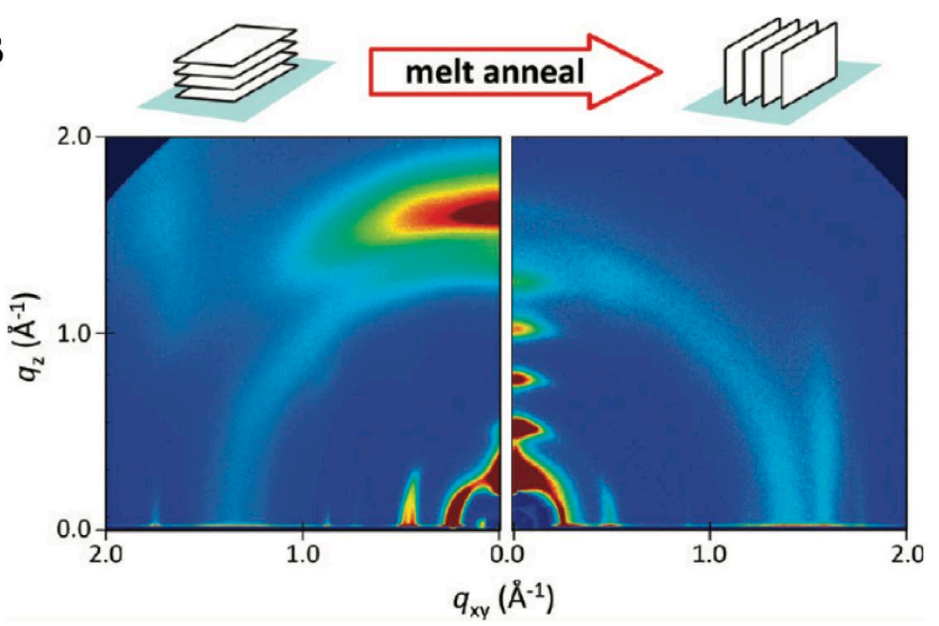

Fig. 6. (A) Schematic illustration of face-on molecular packing of P12 inferred from X-ray data (top) indicating the repeat directions referenced. Proposed microstructural arrangement of the crystallites (bottom) indicating slight disorder in the $\pi$-stacking and lamella stacking directions of the flat, platelet-like crystallites and alignment of the chain backbone direction with the substrate. The complete, branched octyl-decyl side chains are omitted from the schematic illustration due to their unknown relative orientation and order. Adapted from ref.[74] with permission. Copyright (C) 2010 Wiley. (B) Schematic illustration of the packing motifs (top) and GIWAX diffraction patterns associated with annealing a film of P12 to the melt and cooling to ambient at a slow rate. Adapted from ref.[78] with permission. Copyright (C) 2011 American Chemical Society. 
It was proposed that the low degree of energetic disorder of P12 is the origin of its high mobility[79]. When low $\mathrm{k}$ gate dielectrics such as CYTOP and polystyrene (PS) were used, the activation energies were determined to be as low as $44 \mathrm{meV}$ and $64 \mathrm{meV}$ respectively, very comparable to that of the r-Re P3HT and PBTTT. Furthermore, the large NDI unit of P12 makes it rather tolerant to disorder, since even the RI-P(NDI2OD-T2) (P13) still exhibits decent charge transport[80, 81]. The difference in OFET mobilities between RR-P(NDI2ODT2) and RI-P(NDI2OD-T2) is only ca. half order of magnitude[81], in striking contrast to that ( $>4$ orders of magnitude) between Re-P3HT and Ra-P3HT. In the case of P13, the introduction of the irregular linkage does not increase intramolecular steric demand of the backbone but instead inhabits $\pi-\pi$ stacking[80]. Furthermore, the LUMO energies seem to be independent of the regioregularity[81]. This is consistent with recent findings that charges are mostly localized on the NDI2OD moiety instead of strong delocalization along the polymer backbone $[82,83]$. In addition, planarity and $\pi-\pi$ stacking distance seem not to play key roles in the performance of P(NDI2OD-T2). For instance, despite an enhanced backbone planarity and smaller $\pi-\pi$ stacking distance being achieved, the electron mobilities of a few newly synthesized polymers $(\mathbf{P 1 4}, \mathbf{P 1 6})$ are lower compared to the reference polymers $(\mathbf{P 1 5}, \mathbf{P 1 2})$ $[84,85]$, indicating the limitations of using molecular formulae as straightforward predictive tools for device performances[24].

Interestingly, P12 shows a great tendency to pre-aggregate in solution, even at very low concentrations [86], and the level of pre-aggregation directly dictates the extent of aggregation in the thin-film and thus the solid-state charge-transport properties. Intriguingly, when confined within an inert polymer matrix, the diluted P12 film exhibited the X-ray diffraction of a highly disordered material, yet maintained nearly the same local aggregation characteristics of a crystalline neat P12 film[87]. This unique feature allows for decoupling the role of short-range order from ordering along the different crystallographic directions on the charge-transport properties of this high-performance semiconducting polymer[87]. Greater details of this work will be highlighted in chapter 4.1 .

\subsection{Diketopyrrolopyrrole-based copolymers}

In recent years, D-A copolymers based on electron-deficient unit DPP have probably attracted the most attention, as indicated by a surge of publications[56]. DPP displays a planar skeleton, facilitating intermolecular interactions through $\pi-\pi$ stacking. The very-narrow band-gap feature endows DPP-based polymers with very promising performance in both OPVs and OFETs. The extended HOMO and LUMO distribution along the backbone, together with D-A hybridization from the alternating $\mathrm{D}$ and A repeating units, are responsible for the optimized molecular orbital energy levels. As a result, DPP-based polymers show a great tendency to display ambipolarity in OFETs. For instance, the first reported copolymer based on DPP and thiophene (P17, Fig. 7) exhibited quasi-balanced mobilities of $\approx 0.1 \mathrm{~cm}^{2} \mathrm{~V}^{-1} \mathrm{~s}^{-1}$ for both holes and electrons[88]. Interestingly, DPP-thiophene copolymer P18 adopting predominantly faceon orientation exhibited similar mobilities, compared to the analogous DPP-bithiophene and DPP-thienothiophene copolymers (P19 and P20) with edge-on orientations[89]. Hereby, P20 has been one of the most prominent demonstration as ambipolar polymer, which exhibits balanced hole and electron mobilities, both exceeding $1 \mathrm{~cm}^{2} \mathrm{~V}^{-1} \mathrm{~s}^{-1}$ [90]. Remarkably, mobilities up to 8.2 has been reported for a copolymer based on DPP and (E)-2-(2-(thiophen2-yl)vinyl)thiophene (TVT) units (P21)[91]. The origin of such high mobility was attributed to the incorporation of the highly $\pi$-extended TVT units which can enhance the polymer coplanarity and extend the area available for intermolecular $\pi-\pi$ stacking. Furthermore, several DPP-based copolymers using different acceptor units, electronically neutral benzene (P22), the weakly accepting benzothiadiazole (P23), and the strongly accepting benzobisthiadiazole (P24, 
P25), exhibited hole mobility of $\approx 0.17-1.1 \mathrm{~cm}^{2} \mathrm{~V}^{-1} \mathrm{~s}^{-1}$. Surprisingly, no $\pi$-stacking can be detected from the films of all three polymers[92], suggesting that, to achieve high mobility, long-range intermolecular order is not necessary. In addition, the replacement of thiophene with selenophene allows for a promising synthetic way forward for high-mobility ambipolar polymers (P26, P27, P28)[93, 94]. Interestingly, the 2D-GIXD study of P26 indicated a bimodal distribution of face-on and edge-on orientations (Fig.8)[93]. The polymer exhibits quasi-balanced hole and electron mobilities of 0.46 and $0.84 \mathrm{~cm}^{2} \mathrm{~V}^{-1} \mathrm{~s}^{-1}$. These observations of face-on and bimodal packing of these DPP-based copolymers resemble the findings for BTbased P11 and NDI2OD-based P12, which suggests that, efficient charge transport does not necessarily rely on the initially assumed edge-on packing. In-depth reviews of DPP-based copolymers can be found in the literature[56].
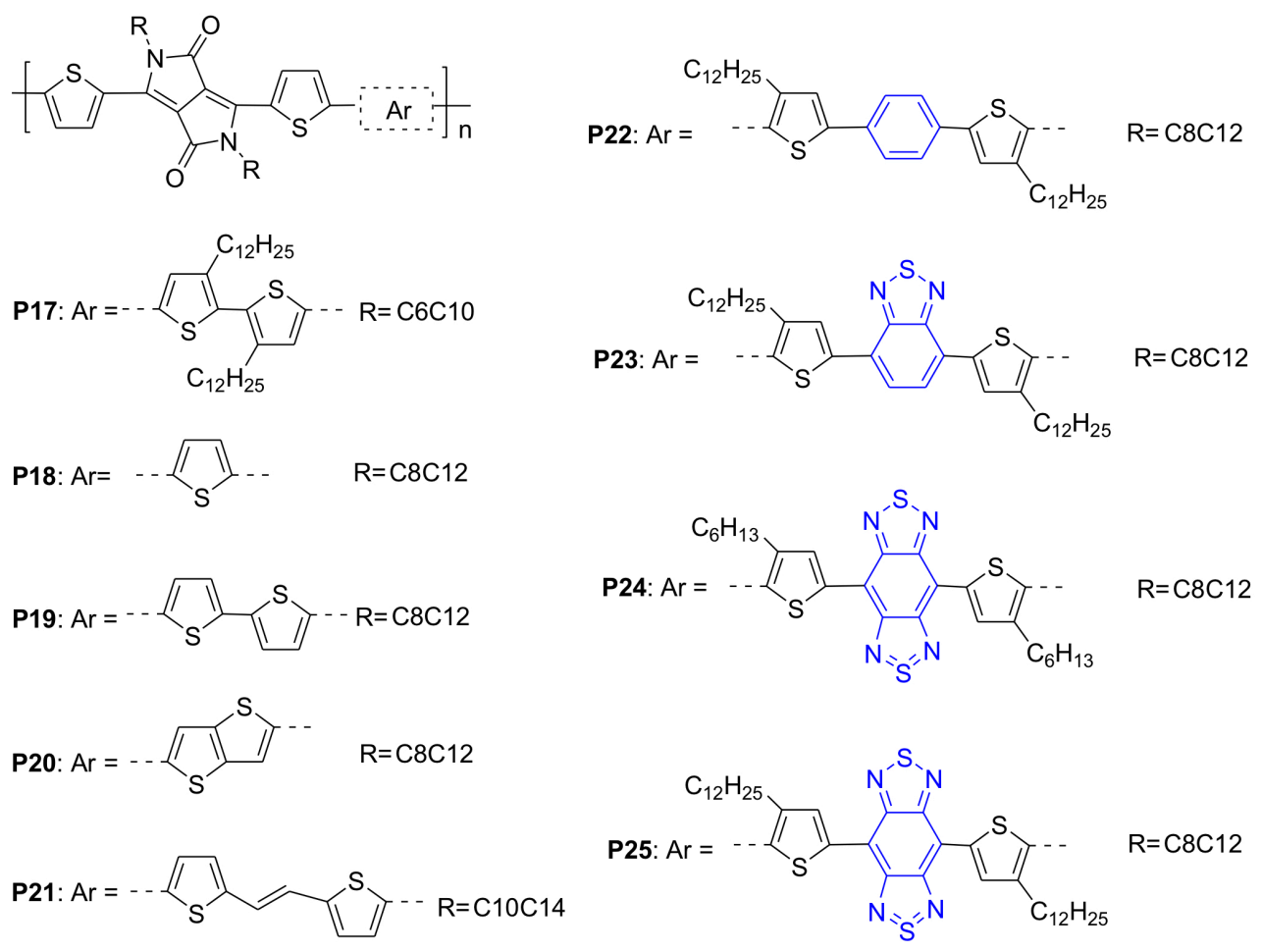

$$
\mathrm{N} \text { "s" } \mathrm{N} \quad \mathrm{C}_{12} \mathrm{H}_{25}
$$
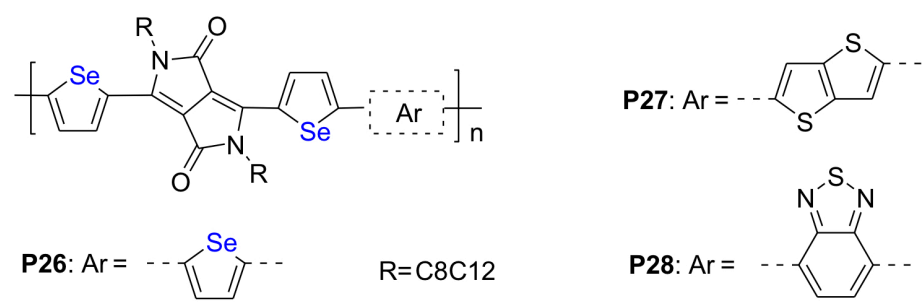

$\mathrm{R}=\mathrm{C} 8 \mathrm{C} 12$

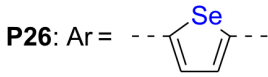

$\mathrm{R}=\mathrm{C} 8 \mathrm{C} 12$

P28: $\mathrm{Ar}=--\backslash$

$\mathrm{R}=\mathrm{C} 8 \mathrm{C} 12$

Fig. 7. Chemical structures of DPP-based copolymers. Acronyms for side chains: C6C10(2hexyl-1-decyl), C8C12(2-octyl-1-dodecyl). 


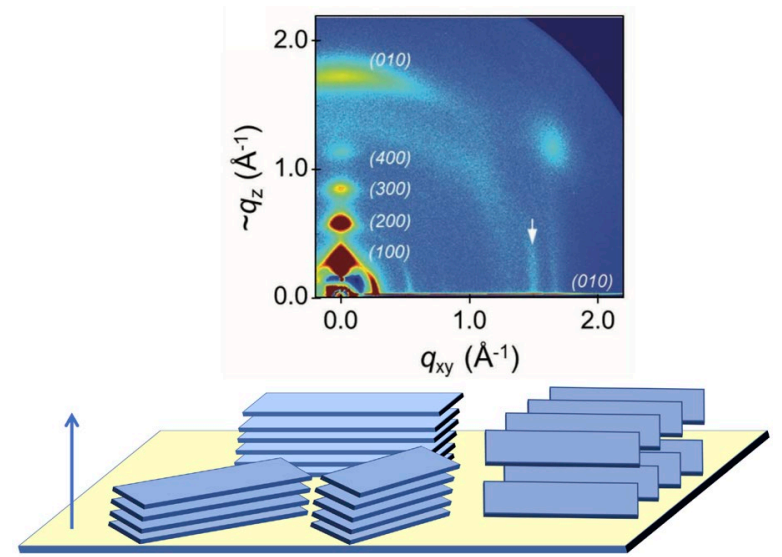

Fig. 8. GIWAX diffraction pattern (top, reprinted from ref.[93] with permission. Copyright $($ ) 2012 Wiley.) and schematic illustration of bi-modal distribution of face-on and edge-on orientations (bottom) of P12 (the blue arrow denotes the film surface normal direction)

\subsection{Isoindigo-based copolymers}

Resembling the emergence of BT-based P1, the initially reported IID-based D-A copolymers highlighted their potential as promising candidates for OPV devices[95, 96]. Later, the use of IID-based copolymers for OFETs has been reported, with mobilities of $\approx 1 \mathrm{~cm}^{2} \mathrm{~V}^{-1} \mathrm{~s}^{-1}$ being achieved. The first report is the IDD-DT(P29, Fig. 9), exhibiting a mobility of $0.79 \mathrm{~cm}^{2} \mathrm{~V}^{-1} \mathrm{~s}^{-}$ ${ }^{1}$ [97]. Intriguingly, $\mathbf{P 2 9}$ is stable in ambient and even high-humidity conditions, ascribed to their low-lying HOMO levels. Remarkably, using a siloxane-terminated solubilizing group as a side chain in an IID-based copolymer P30, a maximum mobility of $\approx 2.5 \mathrm{~cm}^{2} \mathrm{~V}^{-1} \mathrm{~s}^{-1}$ was obtained, in contrast with the reference polymer which has a branched alkyl side chain yielding a maximum mobility of $0.57 \mathrm{~cm}^{2} \mathrm{~V}^{-1} \mathrm{~s}^{-1}[59]$. This was attributed to the smaller $\pi-\pi$ stacking distance of $3.58 \AA$ compared to that of $3.76 \AA$ for the reference polymer. In similar fashion, by careful tailoring alkyl chain branching positions on the polymer backbones, a IID-based copolymer with 4-decyltetradecyl chains (P31) exhibited an even more impressive mobility of $3.6 \mathrm{~cm}^{2} \mathrm{~V}^{-1} \mathrm{~s}^{-1}[98]$, most likely arising from more exposed IID core and tighter intermolecular packing with $\pi-\pi$ stacking distances of $3.57 \AA$. The aforementioned side-chains engineering of BT-based, DPP-based and IID-based copolymers did suggest that, the roles of side chains are far beyond as solubilizing groups, allowing for the tuning a polymer's molecular packing, charge transport, and various physical properties[99]. Strikingly, the first ambipolar IID-based D-A copolymer P32 was reported, which is based on fluorinated IID[100], exhibited mobilities of $0.43 \mathrm{~cm}^{2} \mathrm{~V}^{-1} \mathrm{~s}^{-1}$ for electrons and $1.85 \mathrm{~cm}^{2} \mathrm{~V}^{-1} \mathrm{~s}^{-1}$ for holes. It was observed that the fluorination on the IID unit effectively reduced the LUMO level of the polymer, which greatly increased the electron mobility while maintaining the high hole mobility. Most remarkably, an ultrahigh mobility of $14.4 \mathrm{~cm}^{2} \mathrm{~V}^{-1} \mathrm{~s}^{-1}$ was obtained for the TFT devices using IID analogous polymer containing thienoisoindigo and naphthalene (P33) with a high-k dielectric poly(vinylidenefluoride-trifluoroethylene) ( $\mathrm{P}(\mathrm{VDF}-\mathrm{TrFE}))[15]$. By far, this has been the highest mobility value reported for polymer-based OFETs without chain alignment. Deeper knowledge of IID-based copolymers can be found in the review article[57]. 

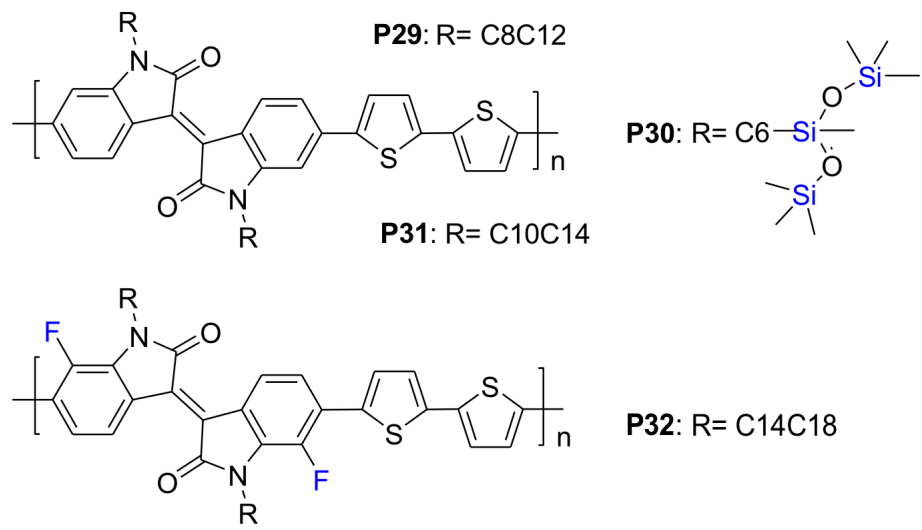

P32: $\mathrm{R}=\mathrm{C} 14 \mathrm{C} 18$

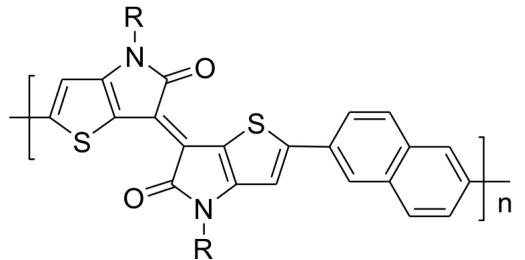

P33: $\mathrm{R}=\mathrm{C} 8 \mathrm{C} 12$

Fig. 9. Chemical structures of DPP-based copolymers. Acronyms for side chains: C8C12(2octyl-1-dodecyl), R(P30)(1,1,1,3,5,5,5-heptamethyltrisiloxane), C10C14(4-decyltetradecyl), C14C18(4-tetradecyl-octadecyl).

\subsection{Some common features of the new host of polymers}

Although having lower level of crystallinity, the emerging high-performance polymers often exhibit similar trap distributions as that of classic highly ordered semicrystalline polymers(Fig. 10)[27]. Theoretically, at a certain level of disorder in the electronic Hamiltonian, the narrower bands present in the new class of conjugated polymers are broadly favorable for charge transport. In other words, the weaker coupling between states can be more than compensated by a lower activation energy for transport[101]. Importantly, these poorly ordered copolymers unanimously display vibronic features in solid-state optical absorption spectra, characteristic of aggregation (defined in this review as a cluster of $\pi$-stacked conjugated segments) [80, 81, $86,92]$. These findings seem to suggest that mobility is, to a greater extent, dictated by the short-range order, rather than the long-range order. Because of their enhanced tolerance to disorder, the same batch of these polymers usually exhibit better cross-lab reproducibility, which is extremely desirable for both research and applications.

Certainly, one cannot disregard what considerable roles that solution processing plays in pushing up the performance of conjugated polymers. Particularly, the most remarkable effect of improving mobility is through polymer chain alignment, simply because the charge transport is fast along the backbone direction, thus it can be significantly blocked if the chains are highly entangled. It is worth highlighting a few examples here. When processed with spin coating[58], and nanogroove[18], the mobilities of $\mathbf{P 1}$ were found to increase from $0.67 \mathrm{~cm}^{2} \mathrm{~V}^{-1} \mathrm{~s}^{-1}$ to 11.4 $\mathrm{cm}^{2} \mathrm{~V}^{-1} \mathrm{~s}^{-1}$, respectively, $\approx 17$ times improvement. More noticeably, the mobilities of $\mathbf{P 8}$ can be pushed to $23.7 \mathrm{~cm}^{2} \mathrm{~V}^{-1} \mathrm{~s}^{-1}$ when processed with combined nano-grooved substrates and slow drying in a tunnel-like configuration, which is about $30 \times$ that of the same polymer processed with drop casting $\left(\mu \approx 0.8 \mathrm{~cm}^{2} \mathrm{~V}^{-1} \mathrm{~s}^{-1}\right)[16]$. Besides, by combining pre-aggregating solvents for preparing the semiconductor and by adopting a room temperature wired bar-coating technique, P12 can exhibit large transport anisotropy $\left(\mu_{\text {parallel }} / \mu_{\text {perpendicular }}>20\right)$ and an electron mobility up to $6.4 \mathrm{~cm}^{2} \mathrm{~V}^{-1} \mathrm{~s}^{-1}$, allowing easy device architectures to operate at $3.3 \mathrm{MHz}[102]$. Comprehensive reviews of advanced solution processing can be found in the literature[10-12, $103,104]$. 


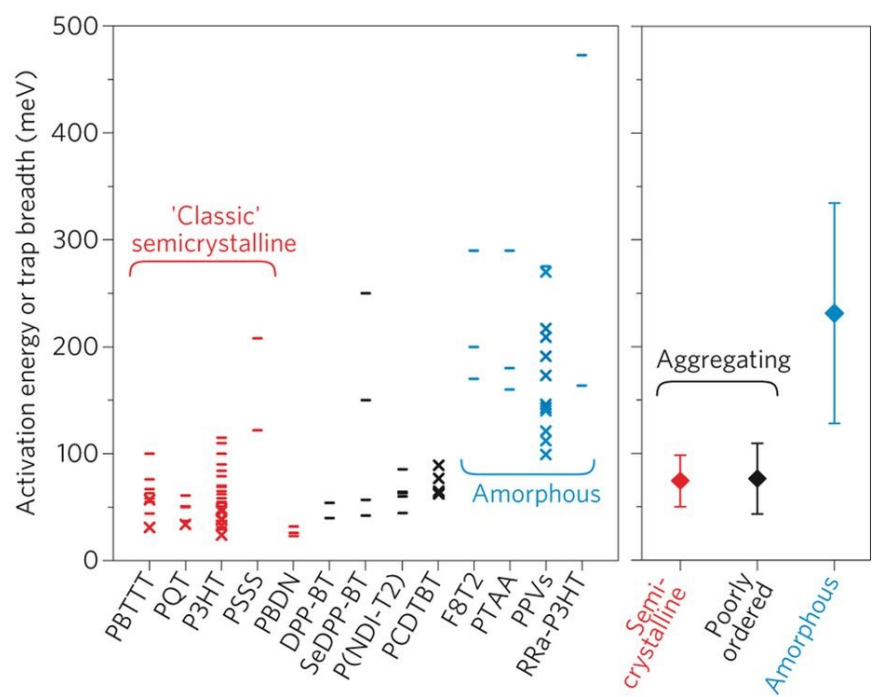

Fig. 10. Activation energies obtained from FET data from Ref. [27], and from the literature (dash), as well as trap depth/tail widths derived from device modelling (cross) for traditional classic semicrystalline materials (red), new high performers that are found to be poorly ordered (black), and completely amorphous materials (blue). Data (84 values for 13 different materials) are binned by material. The averages and standard deviations from the respective groups are shown for semicrystalline, poorly ordered, and amorphous materials on the right panel. Reprinted with permission from Ref.[27]. Copyright (C) 2013, Macmillan Publishers Ltd.

Since the future processing of conjugated polymer is closely related to fast-drying techniques such as ink-printing and roll-to-roll fabrication, it is therefore important to directly evaluate the performance of conjugated polymers without special treatment such as chain alignment. Accordingly, in this review the conjugated polymers were simply processed with common solvents and conventional casting techniques, unless specified otherwise.

\section{Emerging proposals for charge-transport models}

\subsection{A quasi-1D charge-transport model}

While GIWAXS has been widely used to identify the microstructure of conjugated polymers, it reflects the molecular orientation only in crystalline domain, due to the limited sensitivity to amorphous region of this technique. As a supplementary technique, near-edge X-ray absorption fine structure (NEXAFS) is element-specific, bond-sensitive, and allows for estimating the average conformation of polymer chains in the entire films consisting crystalline and noncrystalline regions[46, 105]. The value of dichroic ratio (R) represents an average chain conformation of films. $\mathrm{R}$ can vary from 0.7 for a fully edge-on plane to -1.0 for a complete flat plane, and 0 indicates a comprehensively random orientation[105]. To shed light on the underlying microstructural origin of the exceptional performance of weakly ordered P11(Fig. 11A), both GIWAXS and NEXAFS were performed to deduce the molecular orientation[70].

2D diffraction image acquired from P11 indicated that a majority of backbones align face-on on the substrate (Fig. 11B). The broad and wide diffraction peaks of P11 suggest a substantial disorder in the out-of-plane direction. Nevertheless, the deduced $\pi$-stacking distance of $3.8 \AA$ indicates that local $\pi$-stacking is not disrupted. To investigate the average orientation of the conjugated plane, NEXAFS spectroscopy was performed by evaluating the $1 \mathrm{~s}-\pi^{*}$ resonance intensity as a function of incidence angle $\left(\Theta_{i}\right)$ of the linearly polarized soft X-ray beam (Fig. 11C). Interestingly, it is found that the $\pi^{*}$ intensity decreases significantly as $\Theta_{\mathrm{i}}$ increases (Fig. 
11D), a trend that is contrary to what has been previously observed for classic edge-on orientated polymers, signifying the preferentially face-on orientation of the P11 backbone. This assumption is validated by the fact that, the figure of merit, $\mathrm{R}$ was calculated to be -0.41 for the top surface and -0.56 for the buried interface of P11 films.

The figure of merit $\left\langle\theta_{\pi}\right\rangle$ GIXD and $\left\langle\theta_{\pi}\right\rangle$ NEXAFs allow for deducing orientation of the conjugated planes between crystallites and the volume average of entire films containing both crystalline and non-crystalline fractions. As shown in Fig. 11E, the overlap between the blue traces $\left(\left\langle\theta_{\pi}\right\rangle_{\text {GIXD }}\right)$ and the highlighted region ( $\left\langle\theta_{\pi}\right\rangle$ NEXAFs) reveals a consistency of conjugated plane orientation between the crystalline and non-crystalline regions, implying a common, overall local molecular order. Such an orientation consistency is ascribed to the rigid backbone of P11, leading to that conjugated planes in P11 are oriented in similar ways regardless of whether they are located in crystalline or non-crystalline regions. By contrast, when fast-dried, non-annealed films of P3HT is analyzed, a lack of overlap between the blue traces and the highlighted region is observed (Fig. 11F), suggesting different orientation between the crystalline and non-crystalline regions, attributable to the intrinsic structural difference between P3HT and P11 that the backbones of P3HT are rigidified only in the crystallites, whereas they are still exposed to torsional disorder in non-crystalline regions.

A
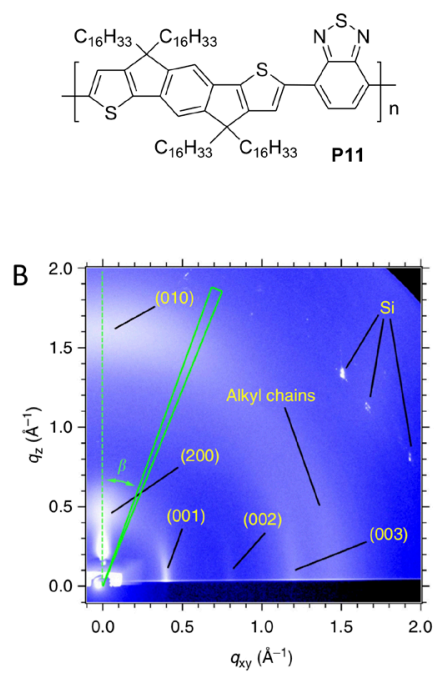

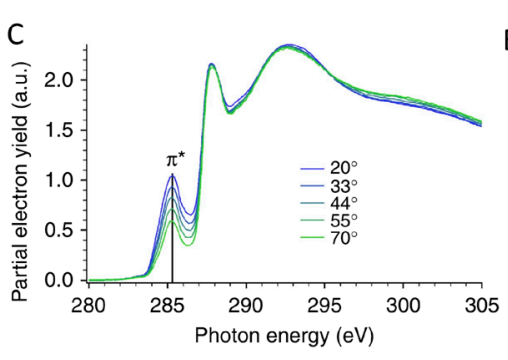

D

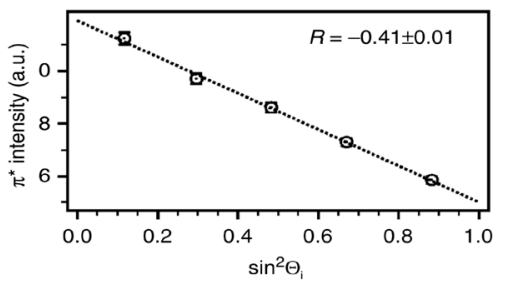

E

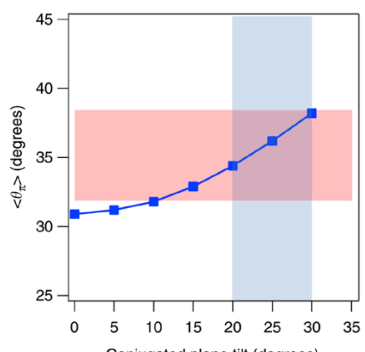

$\mathrm{F}$

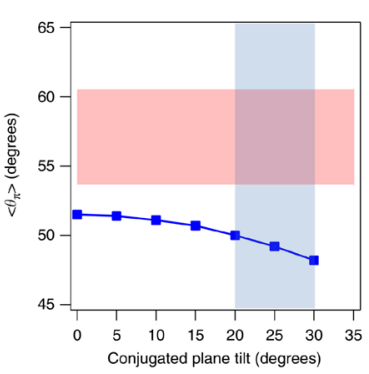

Fig. 11. (A) Chemical structures of P11 with side chains clearly indicated on the molecular structure. (B) 2D-diffraction profile of P11. (C) NEXAFS spectra recorded at five incidence angles $\left(\Theta_{\mathrm{i}}\right)$ for the top surface of IDT-BT films. (D) The $\pi^{*}$ intensity as a function of $\sin ^{2} \Theta_{\mathrm{i}}$ is well fitted into a straight line. NEXAFS partial electron yield and photon energy have standard uncertainties of $\pm 2 \%$ and $\pm 0.1 \mathrm{eV}$, respectively. The average orientation of the conjugated plane calculated from GIXD ( $\left\langle\theta_{\pi}\right\rangle$ GIXD) as a function of the angle between the $(010)$ reflection and the conjugated plane normal for (E) P11, (F) fast-dried, non-annealed P3HT. The upper and lower bounds of the pink areas in e denote the average orientation of the conjugated plane based on the corrected NEXAFS spectra ( $\left\langle\theta_{\pi}\right\rangle$ NEXAFS) of top and buried interfaces, respectively. The pink area in $\mathrm{f}$ is artificially broadened in the vertical direction to match that of e, because P3HT exhibits smaller differences between top and buried interfaces in NEXAFS. The light blue areas highlight the conjugated plane tilt range between $20^{\circ}$ and $30^{\circ}$, that is, the most probable conjugated plane tilt range for alkylated polythiophenes. Adapted from ref.[70] with permission. Copyright (C) 2013, Macmillan Publishers Ltd. 
These results point to the fact that local order is pervasive in the overall microstructures, allowing efficient intermolecular charge transport. The rigid and planar backbones can effectively bridge these locally $\pi$-stacked aggregates, offering an uninterrupted path for charge transport. As a result, quasi-1D charge-transport model has been proposed for the first time, that charge transport in high-mobility semiconducting polymers is mainly occurring along the backbone, with only intermittent $\pi$ linkages to adjacent chains, suggesting the importance of short-range order. Accordingly, the ability to form aggregates seems to be a prerequisite to achieve high mobility for low-crystalline conjugated polymers.

\subsection{A unified charge-transport model: short-range intermolecular aggregation is sufficient for efficient long-range charge transport}

\subsubsection{Structural properties of aggregates govern charge transport}

Recently, the important role of aggregates in charge transport was experimentally studied by measuring the electroluminescence (EL) of blends of Ra-P3HT/ Re-P3HT under various current densities[27]. Interestingly, at current densities comparable to those experienced in electronic devices, the EL spectra of the Ra-P3HT/ Re-P3HT (even down to ratios as low as $10 \%$ Re-P3HT) significantly resemble the spectra of neat RR-P3HT. These results suggest that the charges remain confined in the ordered regions of the film. Therefore, the ordered region, even in short-range (aggregation), is a crucial factor that governs the charge transport in polymer films.

On the other hand, the amorphous fraction does segregate the ordered domains, resulting in poor mobilities, especially for the low-molecular-weight conjugated polymers. However, as long as the molecular weight is high enough, the polymer chains can bridge these aggregates and act as tie-molecules[106]. Experimentally it is suggested that efficient charge transport in such films occurs via connected networks of crystallites[107]. Previous local mobility measurements in high-molecular-weight P3HT indicated that mobility is actually limited by charge transport in nanoscale aggregates, rather than by the electrical connection between these aggregates[108]. This observation is also in agreement with the theoretical modelling that the rate-limiting step is the intermolecular hopping[37]. In addition, scanning-probe field-effect measurements seem to suggest the same conclusion. A transistor based on mobile conductive atomic force microscopy (CAFM) tip was used to probe the nanoscale electrical properties of poly(3-butylthiophene) (P3BT) nanofibers(NFs)[109]. It is observed that the bridging of $\geq 2$ NFs does not reduce the charge transport process in a web of P3BT NFs, and the mobility of one nanofiber is very similar to the mobility for a web of NFs.

\subsubsection{Unifying classic semicrystalline polymers and emerging poorly ordered high performing polymers}

Due to their complex microstructures with various degrees of order, it is difficult to expressly classify conjugated polymers according to their crystalline structures. The introduction of paracrystallinity $[110,111]$ into semiconducting polymers allows a simplification of the ranking of conjugated polymers[112]. Herein, the paracrystallinity parameter $g$ is measured via XRD peak shape analysis, and defined as standard deviation of local, static (cumulative) lattice fluctuations normalized by the average value of the lattice spacing. The equation for calculating the g number is shown in Fig.12A[110, 111]. For all materials, g typically spreads from $0 \%$ to $15 \%$, with $<1 \%$ representing highly crystalline materials, $>10 \%$ representing amorphous materials, such as glass or melt, and $1 \%-10 \%$ representing a paracrystalline materials $[110-$ $112]$. 


$$
{ }^{\mathrm{A}} \quad g_{h k l}=\left(\overline{d_{h k l}^{2}} / \bar{d}_{h k l}^{2}-1\right)^{1 / 2}
$$
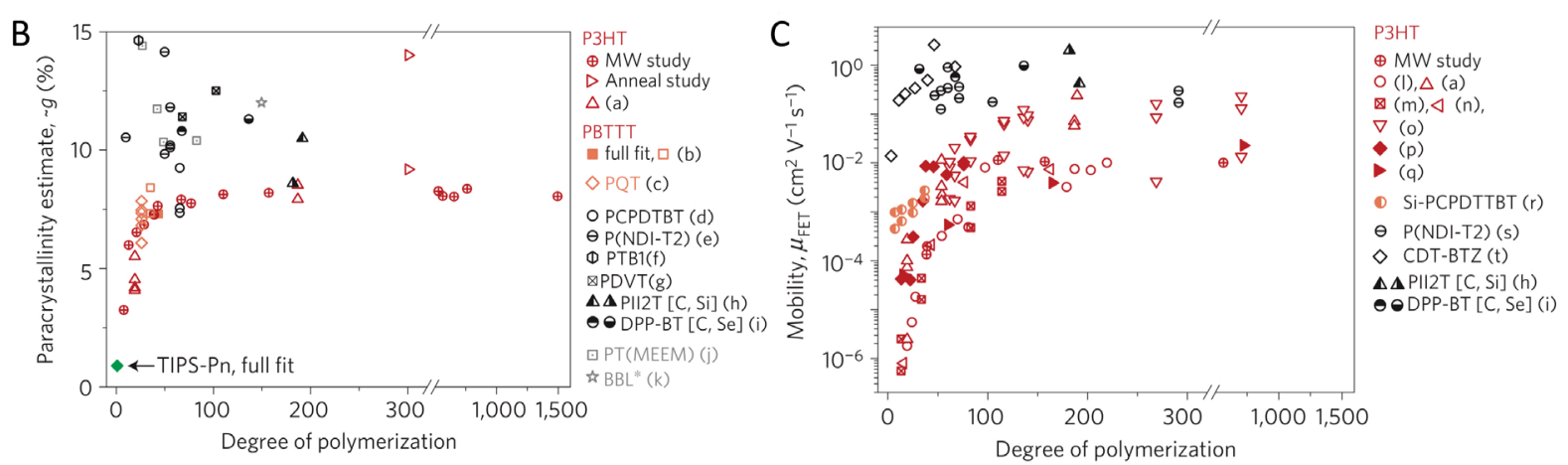

Fig. 12. (A) The equation for calculating the $g$ factor, with $d_{h k l}$ the interplanar separation, and $h, k, l$, the Miller indices. (B)The $\pi$-stacking paracrystallinity in films of polymeric semiconductors of different molecular weight, plotted as a function of degree of polymerization. Semicrystalline materials (P3HT, PBTTT, PQT) are shown in shades of red. Poorly ordered high performing materials are shown in black. Others are shown in grey. Open symbols are estimates, solid symbols are derived from a full analysis. (C) Mobility as a function of molecular weight for a variety of semiconducting polymers. P3HT is shown in red with different symbols referring to different studies. Adapted from ref.[27] with permission. Copyright 2013, Macmillan Publishers Ltd.

By bringing together $\pi$-stacking data from over 30 literature cases, the authors found several striking effects of molecular weight on disorder and on mobility[27]. Firstly, $\pi$-stacking paracrystallinity initially increases with incremental molecular weight and finally becomes independent of molecule length (Fig. 12B). It is surprising that all high-mobility highmolecular-weight polymers show a strong similarity: the $\pi$-stacking paracrystalline disorder is consistently high $(\approx 7 \%-15 \%)$, closer to being amorphous $(>10 \%)$ than being crystalline $(<1 \%)$. Another similarity is the comprehensive trend for the evolution of mobility (firstly increases and then plateaus) as function of molecular weight for all the polymers (Fig. 12C). The longer polymer chains establish a connection between the aggregates and function as tiemolecules[106]. These observations suggest a trade-off between providing electrical connectivity for ordered regions and increasing structural disorder. Taken together, it seems that for low-molecular-weight polymers, the charge transport is connectivity-limited, whereas for high-molecular-weight polymers, the charge transport is lattice-disorder-limited.

Finally, both classic semicrystalline polymers and emerging poorly ordered high-performance polymers can be unified and classified as the same class, where disorder in the intermolecular $\pi$-stacking direction is inevitably high and affects charge transport. As calculated previously, mean free path of charges in the $\pi$-stacks is as short as $\sim 1 \mathrm{~nm}[113]$. Thus, extended crystallites longer than such mean free path will not bring further excellence for charge transport. As a result, in the poorly ordered high performing polymers, short-range aggregation is sufficient for maintaining efficient intermolecular charge transfer and extended crystallinity is not essential. Accordingly, the implications for high-mobility conjugated polymers have been proposed: the key is not to increase their crystallinity but rather to improve their tolerance to an inevitably large amount of disorder. This can be fulfilled by rational design of the polymers allowing more efficient on-chain(by increasing molecular weight) and inter-chain(by designing materials with orbitals having larger overlaps or favorable symmetries) charge-transport 
pathways[27]. Related molecular-design strategies can be found in some recent reviews[99, $114,115]$.

\subsection{A disorder-free charge-transport model: bridging aggregates with torsion-free tie- molecules}

As aforementioned, high molecular weight is considered to be a prerequisite for achieving high mobility, due to the necessity for connectivity of ordered regions. Consequently, recently reported conjugated polymers have prevalently taken molecular mass into consideration. For high-molecular-weight polymers, one of the most important parameters is their persistence length $\left(l_{p}\right)[37,116-120]$, which describes the stiffness of backbone segments. The importance of large persistence lengths for improved transport properties has been highlighted by a coarse grained models[121]. It is suggested that as long as intermolecular charge transport can be ensured by forming aggregations, the increase of persistence length (rigidity) will facilitate better mobility since charges can travel less interruptedly on a single chain, reducing the frequency of rate-limited intermolecular hop. Intuitively, stiffer chains should be able to promote better intramolecular charge delocalization and better bridging the locally $\pi$-stacked aggregates.
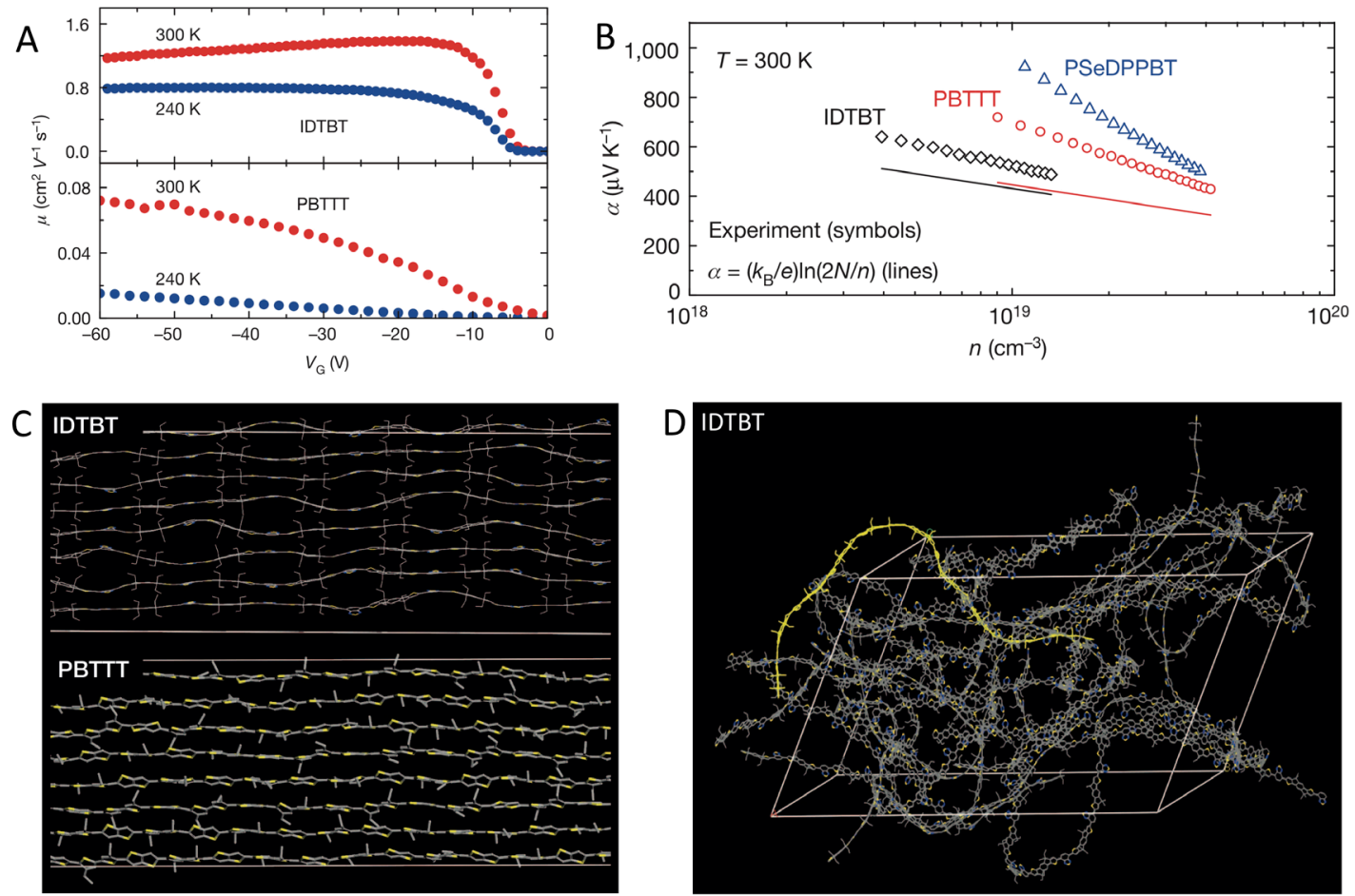

Fig. 13. (A) Gate-voltage dependence of saturation mobility $\mu$ at 300 and $240 \mathrm{~K}$ for patterned P11 (top) and PBTTT (bottom) devices. (B) Slopes of the Seebeck coefficients versus the logarithm of carrier concentration in the accumulation region for P11, PBTTT and P26 at 300 K. The solid lines are plots of $\alpha=(\mathrm{kB} / \mathrm{e}) \ln (2 \mathrm{~N} / \mathrm{n})$. (C) Simulations of the backbone conformation of P11 and PBTTT in side-chain-disordered and non-interdigitated structures. The side chains and hydrogen atoms are omitted for clarity. Yellow, sulphur atoms; blue, nitrogen atoms. (D) Simulation of the backbone conformation of P11 in the amorphous phase. A single chain from the simulated unit cell has been highlighted in bright yellow (other colors as in C). Adapted from ref.[71] with permission. Copyright (C) 2014, Macmillan Publishers Ltd.

Recently, the key role of backbone rigidity was experimentally emphasized via comparatively investigating P11 and PBTTT by temperature-dependent transistor measurements and field- 
effect-modulated Seebeck measurements[71]. Specifically, in the temperature-dependent transistor measurements, the mobility of $\mathbf{P 1 1}$ was found to be nearly invariant when varying gate voltage for $\left|\mathrm{V}_{\mathrm{G}}\right|>20 \mathrm{~V}$ across the entire temperature range (Fig. 13A, top), suggesting very low energetic disorder in P11. By contrast, in PBTTT the mobility significantly increases with gate voltage (Fig. 13A, bottom). These results indicate that energetic disorder is significantly lower in near-amorphous P11 than in highly crystalline PBTTT, which is rather unexpected. Moreover, field-effect-modulated Seebeck measurements, which combines charge and entropy measurements, allow for shedding light on the nature and dynamics of charge transport in organic materials[122, 123]. Fig. 13B shows the field-effect-modulated Seebeck coefficient $(\alpha)$ of holes at room temperature in P11, in comparison to PBTTT and DPP-based copolymer P26. Hereby, it worth mentioning that, for small carrier concentration, the primary contribution to $\alpha$ is the entropy of mixing accompanying adding a carrier into the density of states (DOS), which is dominated by the density of thermally accessible transport states[71]. As a result, in theory, a plot of $\alpha$ versus the logarithm of the mobile carrier density $(\alpha-\log (n))$ yielded a straight line with slope $-(\mathrm{kB} / \mathrm{e}) \ln (10)=-198 \mu \mathrm{V} \mathrm{K}^{-1}$ decade $^{-1}$ (solid lines in Fig. 13B ). Interestingly, the experimental slope of $\alpha-\log (n)$ is the smallest for P11, compared to PBTTT and P26, as shown in Fig. 13B, implying significantly less trapping in P11 than in other two polymers. Note that the mismatch between the experimental and the theoretical slopes is reconciled by considering that a fraction (f) of the charge carriers(n) are trapped in shallow traps and do not get involved in transport. Values of $\mathrm{f}$ were determined to be $0.3,0.5$ and 0.7 for P11, PBTTT and P26, respectively. Together, temperature-dependent transistor measurements and field-effect-modulated Seebeck measurements indicate that all molecular sites are thermally accessible in P11, approaching a disorder-free transport regime[71].

Molecular dynamics (MD) simulations helped further shed light on the molecular origin of the aforementioned disorder-free charge transport of P11. As shown in Fig. 13C, P11 exhibits a wavy, yet tremendously planar and torsion-free backbone, with exceptionally small deviation from planarity (torsion angle of $5.2 \pm 4.0^{\circ}$ ). Such a rigid polymer chain resembles graphene nanoribbons, in which the electrons can move rapidly along the planar chain, despite its lower speed with respect to graphene[124, 125]. By contrast, PBTTT chains spread a broader range of torsion angles $\left(27.2 \pm 14.6^{\circ}\right.$ between T and TT) in spite of its linear conformation (Fig. 13C, bottom). Remarkably enough, even in a completely amorphous phase, IDTBT still accommodates side-chain disorder through bends in the backbone while retaining its nearplanar conformation, (Fig. 13D). Moreover, its DOS is not notably broadened. In contrast, PBTTT single chain displays conformations with larger spans in torsion angles in the amorphous phase and wider DOSs. Convincingly, these MD simulations are well corroborated by the calculations of persistence length, where for P11 it was determined to be as long as $l_{p} \approx$ $29 \mathrm{~nm}[120]$, whereas for PBTTT it was calculated to be $l_{p} \approx 9 \mathrm{~nm}[119]$.

Correspondingly, the implications for 'disorder-free' conjugated polymers have been suggested: (1) minimize the number of torsion-susceptible linkages; (2) planar structure; and (3) long side-chain substitution on both sides of one of the conjugated units to enable space filling in non-interdigitated structures, without disrupting chain rigidity and intermolecular coupling[71]. This work opened up a new avenue for achieving unhampered charge transport and faster organic electronics, despite that much work remains in optimizing the fine balance between intramolecular and intermolecular interactions, to yield the largest overall chargetransfer integrals.

\section{Recent experimental progress based on the role of short-range aggregation}




\subsection{Explicit experimental evidence decoupling the crucial role of short-range order}

Although it has been demonstrated that high charge carrier mobility can be maintained despite the absence of long-range order, there is still a lack of direct experimental evidence which can independently control the long-range order and short-range order and thus can unambiguously clarify their corresponding roles. To this end, we recently reported the successful tuning the degree of long-range order in the polymer while keeping the short-range order integrated, which is based on a nanoconfinement methodology[87]. In this study, $\mathrm{n}$ type aggregating polymer P12 was chosen as model semiconducting polymer (Fig. 14A) and 1,2dichlorobenzene was chosen as solvent which can induce a high degree of preaggregation in the films ( $>30 \%$ of the P12) total mass)[80]. More specifically, by confining the high mobility polymer P12 into extremely thin layers by an inert PS (Fig. 14B) matrix, it is possible to inhibit the $\mathbf{P 1 2}$ chains from crystallizing along $\pi$-stacking or alkyl stacking directions(long-range), so that X-ray diffraction arisen from periodicity in these directions can be suppressed.

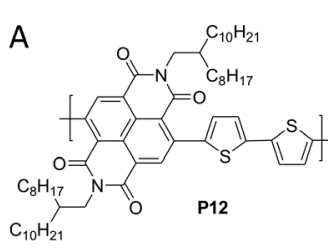

B
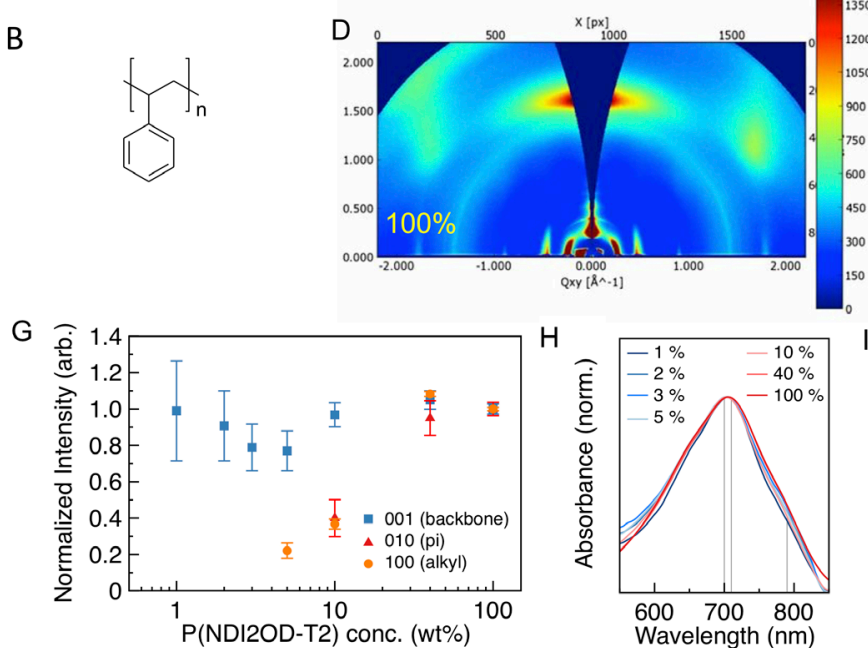
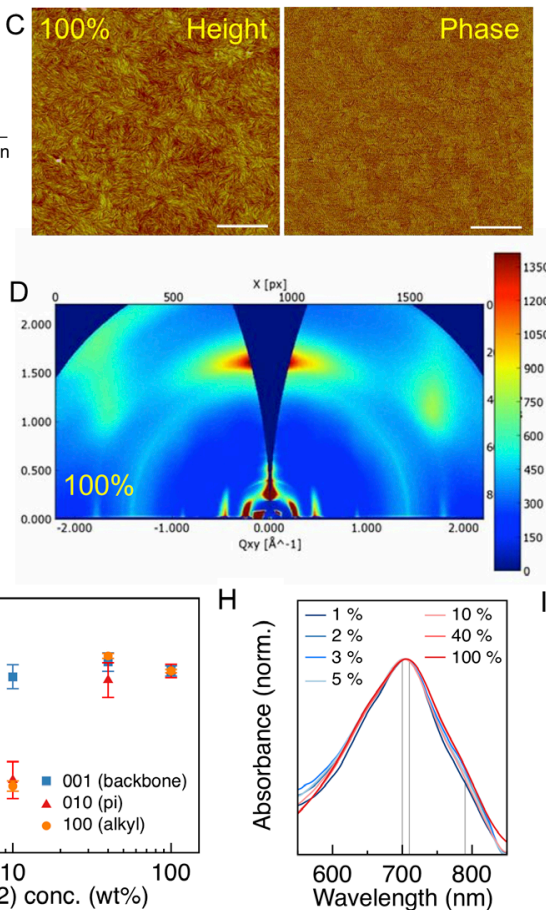
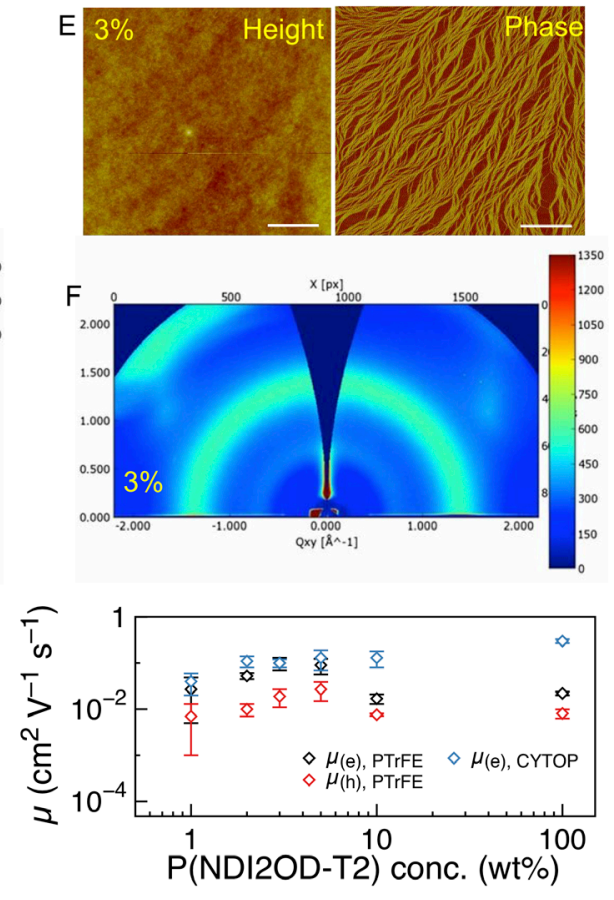

Fig. 14. Chemical structures of (A) P12 with side chains clearly indicated on the molecular structure. and (B) PS. Tapping-mode AFM images of P12/PS films with blend ratios of (C) $100 \%$ (neat) P12, (E) $3 \%$ P12 (left: height; right: phase.), scale bar is $1 \mu \mathrm{m}$. XRD Scattering patterns of (D) neat P12 and (F) $3 \%$ P12. (G) Normalized diffraction intensity of P12 peaks along various crystallographic directions versus P12/PS blend ratio. Intensities have been normalized for both volume of $\mathbf{P 1 2}$ in the film as well as X-ray exposure time. (H) Normalized UV-visible absorption spectra of P12/PS films with different blend ratios. The gray lines indicate the absorption peaks of the aggregates at $\sim 710$ and $\sim 790 \mathrm{~nm}$. (I) Dependence of the electron/hole mobility (calculated at $\left|V_{\mathrm{DS}}\right|=60 \mathrm{~V}$ and normalized for the coverage area, as extracted by AFM image thresholding method) on the P12/PS blend ratio. Reproduced from Ref.[87] with permission. Copyright (C) 2015 the National Academy of Sciences.

As shown in Fig. 14C, an accustomed interconnected, fibrous morphology (Fig. 14C left) featured by single-phase (Fig. 14C right) is observed in neat films of P12. GIWAXS measurements reveal the characteristic diffraction peaks corresponding to the alkyl-stacking (h00), $\pi$-stacking (0k0), and chain backbone (001) directions in neat films of P12 (Fig. 14D). Interestingly, when blending P12 with PS, vertical phase-separation is formed, with most of 
the P12 being accumulated at the topmost part of the film. Fig. 14E is an example of phaseseparated structures at a P12 concentration of $3 \%$, with extended P12 fibers stretching over micrometer-long distances, resembling the films that was previously revealed for neat $\mathbf{P 1 2}$ by high-resolution transmission electron microscopy[126]. A lack of pronounced long-range crystallinity is indicated by the diffuse ring from the GIWAXS image (Fig. 14F). Interestingly, when tracking the order along the various crystallographic directions as a function of the blend ratio, we found different influences of confinement on different crystallographic directions. As shown in Fig. 14G, upon confinement, the long-range packing of the chains is significantly disrupted, as the normalized alkyl- and $\pi$-stacking intensities drop off significantly at a P12 concentration of $10 \%$, and finally completely disappear in films below a P12 concentration of $5 \%$. In a sharp contrast, normalized diffraction intensities arising from the chain backbone remain uninterrupted across all blend films, even in films containing only $1 \%$ P12. These results agree with the long-range organization exhibited in the AFM phase image, that polymer backbones extend over micrometer length-scales. In general, the long-order are disrupted in the dilute films $(<5 \% \mathbf{P 1 2})$.

The optical properties of neat P12 film and P12/PS blended films were investigated by UVVis-NIR absorption spectroscopy (Fig. 14H). The spectrum of all P12/PS blended films (even at $1 \% \mathbf{P 1 2}$ ) is featured by a main absorption band at $\sim 710 \mathrm{~nm}$ and a shoulder at $\sim 790 \mathrm{~nm}$, an evident spectroscopic fingerprint of the formation of aggregations. Interestingly, both diluted and neat P12 film display a quasi-identical low-energy vibronic progress, suggesting the same degree of aggregation in both scenarios. It is worth noting that the low-energy band ascribed to aggregates is governed by the length of straight-chain segments[80]. Therefore, the observed uniform spectrum for all the films demonstrates that the single chain conformation of P12 in both concentrated and dilute films is not far-off, which can be corroborated by the nearly unchanged backbone (001) coherence length of films from $100 \%$ down to $1 \% \mathbf{P 1 2}$.

The charge-transport properties of neat P12 film and P12/PS blended films were investigated by fabricating top-gate OFETs. The mobility is found to be unchanged, irrespective of the P12/PS blended ratios (Fig. 14I). This trend holds true both when high-k polymer and when low-k polymers are separately used as then dielectric insulator. Although the nanoconfinement methodology has been reported previously[127-129], here we make use of these blends as an ideal tool to independently control the degree of long-range and short-range order, and provide unique experimental evidence that short-range intermolecular aggregation is adequate for fast charge transport in conjugated polymers. Finally, the complementary inverters based on these dilute films ( $2 \%-5 \% \mathbf{P 1 2})$ have been realized, exhibiting large gains ( $\approx 65$ for $3 \% \mathbf{P 1 2})$, among the best ever reported for solution-processed organic CMOS-like inverters. Taken together, these results demonstrate that, although the long-range order is disrupted in the dilute films ( $<$ 5\% P12), the P12 chains remain extended and they are interconnected via short-range local aggregation. As a result, these dilute films are able to maintain a high mobility, despite the lack of long-range crystallographic order, at a much lower cost.

This work allows for a clarification of the role of short-range order and connectivity of domains, providing an explanation for the puzzling high mobilities previously observed in the lowcrystalline conjugated polymers, especially those failing to display detectable diffraction in either $\pi$-stacking or alkyl stacking directions $[53,92,130]$ : the aggregations were there, but most probably consisting too few chains so that they were not diffracting. Later, a similar effect of nanoconfinement was reported, where dilution of P3HT in PS matrix leads to a severe disruption of long-range order but results in equivalent or superior mobility, in contrast to neat P3HT[131]. Similarly, a lack of correlation was observed between the crystallinity and the 
charge-transport properties of $\mathrm{P} 3 \mathrm{HT} /$ perylene dicarboximidesn blends, and the only ambipolar charge transport was observed for the blend with lowest crystallinity. Instead, the charge transport was found to be more related to the interconnectivity of domains[132]. In another study, a printing method was used to realize direct aggregation control of conjugated polymers, and the results indicated a close correlation between efficient charge transport and aggregation [133].

Recently, nanoconfinement was successfully used to fabricate flexible electronics based on conjugated-polymer/elastomer, which exhibited efficient charge transport, even when stretched to twice their original length. The confined NFs manifest significantly reduced crystallinity, but still maintain high aggregation. Favorable charge transport is maintained thanks to the connectivity between the nanofibril aggregates[134]. More recently, nanoconfinement was proven to be a practical strategy to fabricate stretchable devices with both improved mechanical elasticity and optical transparency, without affecting the film's electronic conductivity[135, 136].

\subsection{Novel synthesis inspired by the role of short-range order}

Inspired by the emerging proposals that local aggregation can increase interchain charge transport, recently a series of polythiophene-based random copolymers were synthesized with the intention of generating these desired local aggregations[137]. In order to determine the effects of structural change on aggregation, crystallinity and the corresponding device performance, r-Re P3HT (Fig. 15A) was used as reference compound for comparison.

As shown in Fig. 15B, the steric hinderance caused by the side chains can be modulated by changing the side chain density. Specifically, the introduction of several successive pendantfree units into the random copolymer chains was expected to reduce the steric hinderance comparing with the homopolymer. RP17, RP25, and RP33 represent random copolymers with thiophene ratios of $17 \%, 25 \%$, and $33 \%$, respectively. To exclude molecular weight effects, P3HT and all RP copolymers were synthesized to have equal Mn and almost identical PDI. DFT calculations reveal the role of thiophene units in the planarization of the polymer backbone. As shown in the model for P3HT (Fig. 15C), the dihedral angles $\Phi 1$ and $\Phi 2$ between three central thiophene rings were estimated to be $33.6^{\circ}$ and $32.9^{\circ}$, respectively. The introduction of thiophene units leads to reduced steric hindrance. As illustrated in Fig. 15D, when the number of thiophene units reached three, a more planar conformation with dramatically decreased of dihedral angles $\left(\Phi 1=19^{\circ}, \Phi 2=17^{\circ}\right)$ was achieved.

The optical properties of P3HT and RPs in solution and annealed films were investigated by UV-vis-NIR absorption spectroscopy. As the thiophene content increasing, absorption spectra exhibited a gradual bathochromic behavior in chloroform, chlorobenzene, and toluene. The red shift indicated an increase in the effective conjugation length of the polymer backbone, which is highly relevant to backbone planarity in solution state. In addition, a clear spectroscopic fingerprint of the formation of aggregate species was evidenced by the shoulder peak at around $600 \mathrm{~nm}$ in the absorption spectrum of annealed films (Fig. 15E). Interestingly, the $0-0 / 0-1$ absorption ratio gradually increased with increasing thiophene content, which can be attributed to the enhanced intrachain exciton coupling induced by the planarization of backbones in RPs. These optical spectra data are well consistent with the DFT modelling of the polymer backbone conformation evolution. 


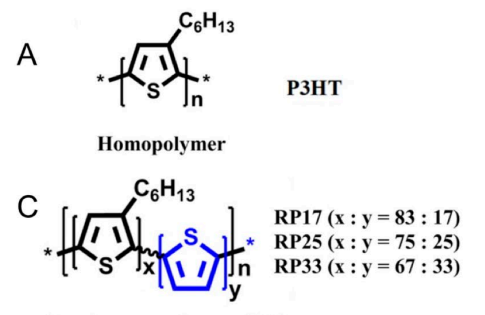

B
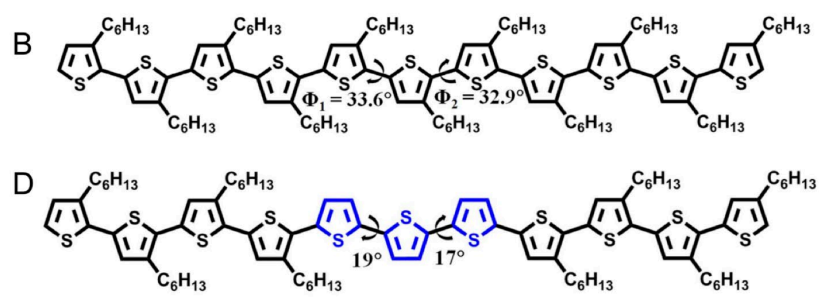

Random copolymer (RP)
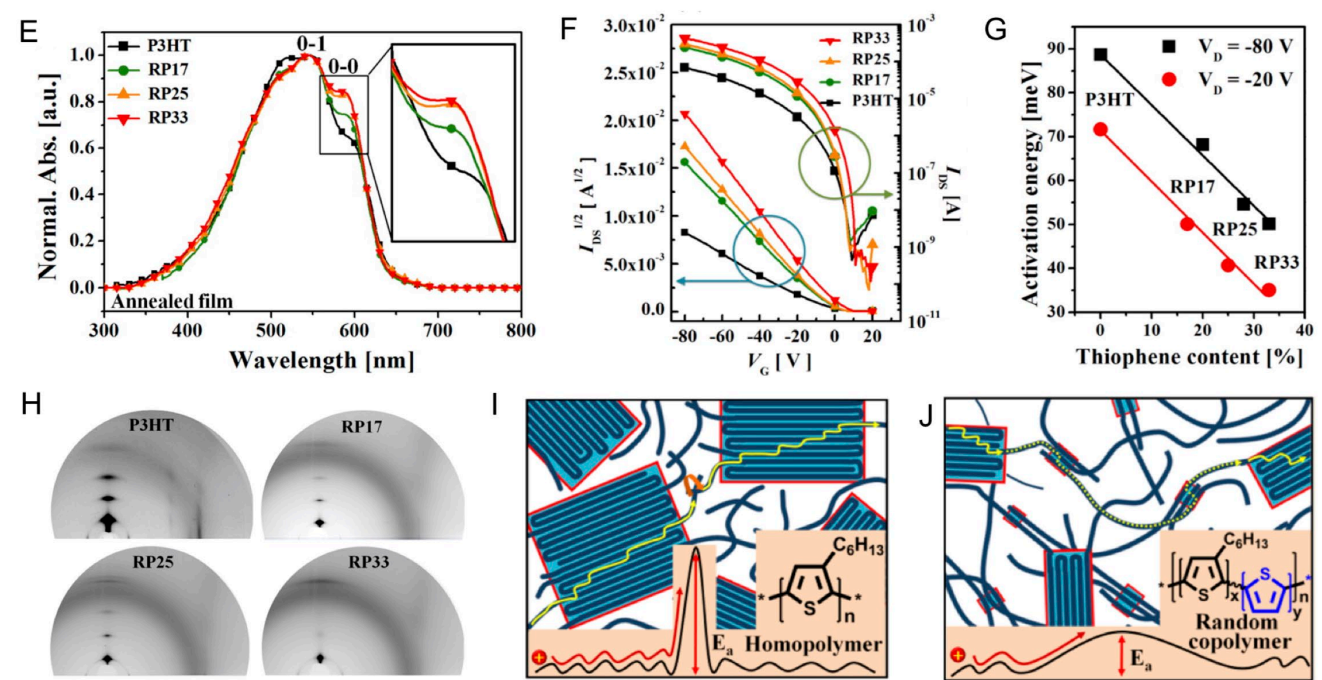

Fig. 15. Chemical structures of (A) P3HT homopolymer, and (C) Random 3-hexylthiophenethiophene copolymers. Chemical structures of the oligothiophenes containing thiophene units of (B) 0 , and (D)3, considered in the DFT calculations and dihedral angles calculated at the B3LYP/6-31G(d,p) level of theory. (E) Normalized UV-vis absorption spectra of the annealed polymer film. (F) Transfer characteristics at a fixed $V_{\text {drain }}\left(V_{D}\right)$ of $-80 \mathrm{~V}$ for OFETs using annealed polymer films. (G)Calculated activation energies at a $V_{D}$ of -80 and $-20 \mathrm{~V}$. (H) Twodimensional GIWAXS patterns of annealed polymer films. Schematic representations of (I) a P3HT-type microstructure exhibiting high crystallinity and poor interconnectivity in amorphous regions and $(\mathrm{J})$ an RP33-type microstructure presenting low crystallinity and great interconnectivity via localized aggregates in amorphous regions. Reproduced from Ref.[137] with permission. Copyright (C) 2016 American Chemical Society.

2D-GIWAXS was performed to investigate the change of the polymer film microstructures with increasing thiophene content. As shown in Fig. 15H, the P3HT film exhibits a typical diffraction pattern which agrees with previous reports on P3HT. As the thiophene content increases, the intensity of both the out-of-plane peaks (h00) and the in-plane (010) peak were reduced, indicating the decrease in film crystallinity. Interestingly, all peak intensities decreased except for the out-of-plane (010) peak related to out-of-plane $\pi-\pi$ stacking. Hereby, RP33 displays the strongest (010) peak, indicating the highest degree of out-of-plane $\pi-\pi$ stacking, which is assumed to originate from local aggregation formed by facilitated $\pi-\pi$ stacking owing to the planarized backbone and the reduced steric hindrance. Furthermore, a smaller $d$-spacings and $\pi-\pi$ stacking distance was observed with increasing thiophene contents. NEXAFS measurements validated the 2D-WAXS results, and confirmed that RPs adopted a general face-on chain orientation, despite the fact that an edge-on orientation was detected in their crystallites. Again, NEXAFS provides the azimuthal averages of unknown orientation distributions, rather than explicit molecular orientations within crystals. 
OFETs allows for a direct assessment on the impact of the thiophene units on the chargetransport properties of the polymers. Interestingly, the higher the thiophene content is introduced, higher OFETs performance can be obtained (Fig. 15F). Remarkably, RP33, the one with the lowest degree of crystallinity, presented the highest mobility of $1.37 \mathrm{~cm}^{2} \mathrm{~V}^{-1} \mathrm{~s}^{-1}$, up to $\approx 7$ times higher than the highly ordered P3HT $\left(\mu=0.19 \mathrm{~cm}^{2} \mathrm{~V}^{-1} \mathrm{~s}^{-1}\right)$. Moreover, both the activation energies measured at the high and low drain voltages decreased linearly with increasing thiophene content (Fig. 15G), indicating that local aggregation formed in RPs facilitates charge transport. Correspondingly, schematic illustrations of the microstructures for P3HT and RPs are shown in Fig. 15I, J. This works clearly demonstrates the relative importance of short-range order and long-range order in the performance of conjugated polymers and set a very practical example for future chemical synthesis[137].

Steady-state absorption and fluorescence spectra demonstrated that increasing the intrachain torsional disorder in aggregates increases the energy and breadth of DOS. By extracting polaron dynamics in the transient absorption spectra, it was observed that an activation energy barrier of $50 \mathrm{meV}$ is imposed on the charge separation process in $\mathrm{P} 3 \mathrm{HT}$, whereas it is essentially barrierless in RP33[138]. Impressively, mechanical properties of RP33, such as elongation at break, tensile strength, and toughness were improved along with charge carrier mobility. Besides, it showed great tolerance to a $40 \%$ strain without a decrease in mobility and maintained a stable electrical performance even after 5000 stretching cycles at 30\% strain[139].

\section{Conclusions and perspectives}

The last three decades have witnessed significant progress in the field of organic electronics, including both materials breakthrough, and enriched understanding of charge transport, thanks to the joint efforts of scientists in multiple disciplines. The first milestone established in the 1990s has led to the 2D charge-transport model and generated a wealth of knowledge, which is still guiding the mainstream of the research in this field. The second milestone built in the late 2000s started from the surprise that charge transport is even more efficient in nearamorphous polymer than that in the highly ordered, crystalline one. With collective efforts of experimentalists and theoreticians, the greater tolerance to disorder exhibited by the nearamorphous polymers seemed to be explained by quasi-1D charge-transport model and unified charge-transport model. Explicit evidence appeared that local aggregation over very few chains is a sufficient mesoscopic structure to ensure high mobility, with charge transport mainly occurring along the polymer backbones, and that extended crystallinity is not indispensable. Particularly, the disorder-free charge-transport model allows maximizing charge-transfer integrals in conjugated polymers, with every molecular unit along the backbone being able to participate in charge transport, representing another important step towards faster organic electronics. Recent experimental progress also suggests a great potential of conjugated polymers in the application of flexible/stretchable organic electronics, owing to their ability of overcoming the trade-off between charge carrier mobility and flexibility/stretchability[140, 141]. Together, these remarkable advances have brought organic electronics community closer to the bright future promised by organic electronics applications.

Despite the aforementioned advances, considerable challenges still exist in this field. For example, the interplay between structure and properties of conjugated polymers remains far from being fully articulated. There is still a lack of multi-length-scale approach that can straightforwardly predict device performance from molecular structure, mainly due to a multitude of variables beyond molecular formulae, e.g. molecular weight, PDI, chain rigidity, planarity, conjugated core size, side chain length and density, heteroatoms, and end-capping 
etc. No single variable is fully responsible for the eventual device performance. Therefore, a lot of work remains in predicting the macroscopic performance from the molecular structure.

Another challenge therefore arises that many empirical molecular design rules may not necessarily apply to all molecular systems, sometimes yielding unexpected charge transport performance[84]. But on the other hand, an unexpected result is not necessarily undesired, which is likely to refine our knowledge, yielding new molecular designing rules, as well as leading to the further evolution of the charge-transport models. Such a process has been happening continuously, especially during the past decade, and it is surely going to happen in the future.

While there are droves of polymers that can outperform amorphous silicon, another longstanding challenge remains: their operational and environmental stability[142, 143], which hampers the commercialization of polymers. To date, very few polymers can be operated under ambient conditions. Again, encouragingly, recent reports demonstrated that the operational and environmental stability could be achieved either by the addition of molecular additives[144] or via locking polymer backbones into a fully rigid conformation[145], bringing further hope in reaching the ultimate goal of organic electronics community.

\section{Acknowledgement}

The author is appreciative of Linköping University for providing him with the current research platform. Over the years, the author has been inspired by many colleagues and co-workers. Herein, the author particularly would like to thank Dr. S. Fabiano, Prof. X. Crispin, Prof. M. Berggren, Prof. W. Pisula, and Prof. K. Müllen, for their help and support.

\section{References}

[1] S.R. Forrest, Nature, 428 (2004) 911-918.

[2] H. Sirringhaus, Advanced Materials, 17 (2005) 2411-2425.

[3] A. Salleo, Materials Today, 10 (2007) 38-45.

[4] M. Berggren, D. Nilsson, N.D. Robinson, Nature Materials, 6 (2007) 3-5.

[5] A.C. Arias, J.D. MacKenzie, I. McCulloch, J. Rivnay, A. Salleo, Chemical Reviews, 110 (2010) 3-24.

[6] A. Facchetti, Chemistry of Materials, 23 (2011) 733-758.

[7] C. Wang, H. Dong, W. Hu, Y. Liu, D. Zhu, Chem Rev, 112 (2012) 2208-2267.

[8] X. Guo, M. Baumgarten, K. Müllen, Progress in Polymer Science, 38 (2013) 1832-1908.

[9] J. Mei, Y. Diao, A.L. Appleton, L. Fang, Z.N. Bao, J Am Chem Soc, 135 (2013) 67246746.

[10] H.N. Tsao, K. Müllen, Chem Soc Rev, 39 (2010) 2372-2386.

[11] Y. Yao, H. Dong, W. Hu, Polymer Chemistry, 4 (2013) 5197-5205.

[12] Y. Diao, L. Shaw, Z.N. Bao, S.C.B. Mannsfeld, Energy \& Environmental Science, 7 (2014) 2145-2159.

[13] J. Li, Y. Zhao, H.S. Tan, Y. Guo, C.A. Di, G. Yu, Y. Liu, M. Lin, S.H. Lim, Y. Zhou, H. Su, B.S. Ong, Sci Rep, 2 (2012) 754.

[14] I. Kang, H.J. Yun, D.S. Chung, S.K. Kwon, Y.H. Kim, Journal of the American Chemical Society, 135 (2013) 14896-14899. 
[15] G. Kim, S.J. Kang, G.K. Dutta, Y.K. Han, T.J. Shin, Y.Y. Noh, C. Yang, Journal of the American Chemical Society, 136 (2014) 9477-9483.

[16] H.R. Tseng, H. Phan, C. Luo, M. Wang, L.A. Perez, S.N. Patel, L. Ying, E.J. Kramer, T.Q. Nguyen, G.C. Bazan, A.J. Heeger, Advanced Materials, 26 (2014) 2993-2998.

[17] C. Luo, A.K.K. Kyaw, L.A. Perez, S. Patel, M. Wang, B. Grimm, G.C. Bazan, E.J. Kramer, A.J. Heeger, Nano Letters, 14 (2014) 2764-2771.

[18] Y. Yamashita, F. Hinkel, T. Marszalek, W. Zajaczkowski, W. Pisula, M. Baumgarten, H. Matsui, K. Müllen, J. Takeya, Chemistry of Materials, 28 (2016) 420-424.

[19] F.J. Lin, C. Guo, W.T. Chuang, C.L. Wang, Q.B. Wang, H. Liu, C.S. Hsu, L. Jiang, Advanced Materials, 29 (2017) 1606987.

[20] J. Lee, S.H. Kang, S.M. Lee, K.C. Lee, H. Yang, Y. Cho, D. Han, Y.F. Li, B.H. Lee, C. Yang, Angewandte Chemie-International Edition, 57 (2018) 13629-13634.

[21] Y. Park, J.W. Jung, H. Kang, J. Seth, Y. Kang, M.M. Sung, Nano Lett, 19 (2019) 10281032.

[22] H.L. Dong, X.L. Fu, J. Liu, Z.R. Wang, W.P. Hu, Advanced Materials, 25 (2013) 61586182.

[23] Y. Olivier, D. Niedzialek, V. Lemaur, W. Pisula, K. Müllen, U. Koldemir, J.R. Reynolds, R. Lazzaroni, J. Cornil, D. Beljonne, Adv Mater, 26 (2014) 2119-2136.

[24] Z.B. Henson, K. Müllen, G.C. Bazan, Nat Chem, 4 (2012) 699-704.

[25] H. Sirringhaus, Advanced Materials, 26 (2014) 1319-1335.

[26] D. Fazzi, M. Caironi, Physical Chemistry Chemical Physics, 17 (2015) 8573-8590.

[27] R. Noriega, J. Rivnay, K. Vandewal, F.P. Koch, N. Stingelin, P. Smith, M.F. Toney, A. Salleo, Nat Mater, 12 (2013) 1038-1044.

[28] A. Assadi, C. Svensson, M. Willander, O. Inganas, Applied Physics Letters, 53 (1988) 195-197.

[29] J. Paloheimo, P. Kuivalainen, H. Stubb, E. Vuorimaa, P. Ylilahti, Applied Physics Letters, 56 (1990) 1157-1159.

[30] P.W.M. Blom, M.J.M. deJong, J.J.M. Vleggaar, Applied Physics Letters, 68 (1996) 33083310.

[31] S. Son, A. Dodabalapur, A.J. Lovinger, M.E. Galvin, Science, 269 (1995) 376-378.

[32] Z.N. Bao, A. Dodabalapur, A.J. Lovinger, Applied Physics Letters, 69 (1996) 4108-4110.

[33] H. Sirringhaus, P.J. Brown, R.H. Friend, M.M. Nielsen, K. Bechgaard, B.M.W. Langeveld-Voss, A.J.H. Spiering, R.A.J. Janssen, E.W. Meijer, P. Herwig, D.M. de Leeuw, Nature, 401 (1999) 685-688.

[34] H. Sirringhaus, N. Tessler, R.H. Friend, Science, 280 (1998) 1741-1744.

[35] H. Sirringhaus, P.J. Brown, R.H. Friend, M.M. Nielsen, K. Bechgaard, B.M.W. Langeveld-Voss, A.J.H. Spiering, R.A.J. Janssen, E.W. Meijer, Synthetic Metals, 111 (2000) 129-132.

[36] A. Devizis, K. Meerholz, D. Hertel, V. Gulbinas, Chemical Physics Letters, 498 (2010) 302-306.

[37] R. Noriega, A. Salleo, A.J. Spakowitz, Proceedings of the National Academy of Sciences of the United States of America, 110 (2013) 16315-16320.

[38] B.S. Ong, Y.L. Wu, P. Liu, S. Gardner, Journal of the American Chemical Society, 126 (2004) 3378-3379.

[39] B.S. Ong, Y.L. Wu, P. Liu, S. Gardner, Advanced Materials, 17 (2005) 1141-1144.

[40] R.J. Kline, D.M. DeLongchamp, D.A. Fischer, E.K. Lin, L.J. Richter, M.L. Chabinyc, M.F. Toney, M. Heeney, I. McCulloch, Macromolecules, 40 (2007) 7960-7965.

[41] I. McCulloch, M. Heeney, C. Bailey, K. Genevicius, I. Macdonald, M. Shkunov, D. Sparrowe, S. Tierney, R. Wagner, W.M. Zhang, M.L. Chabinyc, R.J. Kline, M.D. McGehee, M.F. Toney, Nature Materials, 5 (2006) 328-333. 
[42] B.H. Hamadani, D.J. Gundlach, I. McCulloch, M. Heeney, Applied Physics Letters, 91 (2007).

[43] S. Malik, A.K. Nandi, Journal of Polymer Science Part B-Polymer Physics, 40 (2002) 2073-2085.

[44] O.F. Pascui, R. Lohwasser, M. Sommer, M. Thelakkat, T. Thurn-Albrecht, K. Saalwachter, Macromolecules, 43 (2010) 9401-9410.

[45] C.R. Snyder, R.C. Nieuwendaal, D.M. DeLongchamp, C.K. Luscombe, P. Sista, S.D. Boyd, Macromolecules, 47 (2014) 3942-3950.

[46] I. McCulloch, M. Heeney, M.L. Chabinyc, D. DeLongchamp, R.J. Kline, M. Coelle, W. Duffy, D. Fischer, D. Gundlach, B. Hamadani, R. Hamilton, L. Richter, A. Salleo, M. Shkunov, D. Sporrowe, S. Tierney, W. Zhong, Advanced Materials, 21 (2009) 1091-1109.

[47] R.J. Kline, M.D. McGehee, E.N. Kadnikova, J.S. Liu, J.M.J. Frechet, M.F. Toney, Macromolecules, 38 (2005) 3312-3319.

[48] S. Himmelberger, K. Vandewal, Z. Fei, M. Heeney, A. Salleo, Macromolecules, 47 (2014) 7151-7157.

[49] X. Zhang, S.D. Hudson, D.M. DeLongchamp, D.J. Gundlach, M. Heeney, I. McCulloch, Advanced Functional Materials, 20 (2010) 4098-4106.

[50] K. Müllen, W. Pisula, Journal of the American Chemical Society, 137 (2015) 9503-9505.

[51] D. Mühlbacher, M. Scharber, M. Morana, Z. Zhu, D. Waller, R. Gaudiana, C. Brabec, Advanced Materials, 18 (2006) 2884-2889.

[52] Z. Zhu, D. Waller, R. Gaudiana, M. Morana, D. Muhlbacher, M. Scharber, C. Brabec, Macromolecules, 40 (2007) 1981-1986.

[53] M. Zhang, H.N. Tsao, W. Pisula, C.D. Yang, A.K. Mishra, K. Müllen, Journal of the American Chemical Society, 129 (2007) 3472-3473.

[54] Z.H. Chen, Y. Zheng, H. Yan, A. Facchetti, Journal of the American Chemical Society, 131 (2009) 8-9.

[55] W.M. Zhang, J. Smith, S.E. Watkins, R. Gysel, M. McGehee, A. Salleo, J. Kirkpatrick, S. Ashraf, T. Anthopoulos, M. Heeney, I. McCulloch, Journal of the American Chemical Society, 132 (2010) 11437-11439.

[56] C.B. Nielsen, M. Turbiez, I. McCulloch, Adv Mater, 25 (2013) 1859-1880.

[57] T. Lei, J.Y. Wang, J. Pei, Accounts of Chemical Research, 47 (2014) 1117-1126.

[58] H.N. Tsao, D. Cho, J.W. Andreasen, A. Rouhanipour, D.W. Breiby, W. Pisula, K. Müllen, Advanced Materials, 21 (2009) 209-212.

[59] J. Mei, D.H. Kim, A.L. Ayzner, M.F. Toney, Z.N. Bao, J Am Chem Soc, 133 (2011) 20130-20133.

[60] H.N. Tsao, D.M. Cho, I. Park, M.R. Hansen, A. Mavrinskiy, D.Y. Yoon, R. Graf, W. Pisula, H.W. Spiess, K. Müllen, Journal of the American Chemical Society, 133 (2011) 2605 2612.

[61] D. Niedzialek, V. Lemaur, D. Dudenko, J. Shu, M.R. Hansen, J.W. Andreasen, W. Pisula, K. Müllen, J. Cornil, D. Beljonne, Adv Mater, 25 (2013) 1939-1947.

[62] Y. Zhang, J.Y. Zou, C.C. Cheuh, H.L. Yip, A.K.Y. Jen, Macromolecules, 45 (2012) $5427-$ 5435.

[63] W. Pisula, H.N. Tsao, D. Dudenko, D.M. Cho, S.R. Puniredd, Y.F. Zhao, A. Mavrinskiy, J. Shu, M.R. Hansen, M. Baumgarten, K. Müllen, Polymers, 5 (2013) 833-846.

[64] P.M. Beaujuge, H.N. Tsao, M.R. Hansen, C.M. Amb, C. Risko, J. Subbiah, K.R. Choudhury, A. Mavrinskiy, W. Pisula, J.L. Bredas, F. So, K. Müllen, J.R. Reynods, Journal of the American Chemical Society, 134 (2012) 8944-8957.

[65] Z.P. Fei, J.S. Kim, J. Smith, E.B. Domingo, T.D. Anthopoulos, N. Stingelin, S.E. Watkins, J.S. Kim, M. Heeney, J Mater Chem, 21 (2011) 16257-16263. 
[66] U. Koldemir, S.R. Puniredd, M. Wagner, S. Tongay, T.D. McCarley, G.D. Kamenov, K. Müllen, W. Pisula, J.R. Reynolds, Macromolecules, 48 (2015) 6369-6377.

[67] L. Ying, B.B.Y. Hsu, H.M. Zhan, G.C. Welch, P. Zalar, L.A. Perez, E.J. Kramer, T.Q. Nguyen, A.J. Heeger, W.Y. Wong, G.C. Bazan, Journal of the American Chemical Society, 133 (2011) 18538-18541.

[68] M.M. Li, C.B. An, W. Pisula, K. Müllen, Accounts of Chemical Research, 51 (2018) 1196-1205.

[69] J. Kirkpatrick, C.B. Nielsen, W.M. Zhang, H. Bronstein, R.S. Ashraf, M. Heeney, I. McCulloch, Advanced Energy Materials, 2 (2012) 260-265.

[70] X. Zhang, H. Bronstein, A.J. Kronemeijer, J. Smith, Y. Kim, R.J. Kline, L.J. Richter, T.D. Anthopoulos, H. Sirringhaus, K. Song, M. Heeney, W. Zhang, I. McCulloch, D.M. DeLongchamp, Nat Commun, 4 (2013) 2238.

[71] D. Venkateshvaran, M. Nikolka, A. Sadhanala, V. Lemaur, M. Zelazny, M. Kepa, M. Hurhangee, A.J. Kronemeijer, V. Pecunia, I. Nasrallah, I. Romanov, K. Broch, I. McCulloch, D. Emin, Y. Olivier, J. Cornil, D. Beljonne, H. Sirringhaus, Nature, 515 (2014) 384-388.

[72] D. Fazzi, M. Caironi, C. Castiglioni, Journal of the American Chemical Society, 133 (2011) 19056-19059.

[73] E. Giussani, D. Fazzi, L. Brambilla, M. Caironi, C. Castiglioni, Macromolecules, 46 (2013) 2658-2670.

[74] J. Rivnay, M.F. Toney, Y. Zheng, I.V. Kauvar, Z.H. Chen, V. Wagner, A. Facchetti, A. Salleo, Advanced Materials, 22 (2010) 4359-4363.

[75] B.A. Collins, J.E. Cochran, H. Yan, E. Gann, C. Hub, R. Fink, C. Wang, T. Schuettfort, C.R. McNeill, M.L. Chabinyc, H. Ade, Nature Materials, 11 (2012) 536-543.

[76] T. Schuettfort, L. Thomsen, C.R. McNeill, Journal of the American Chemical Society, 135 (2013) 1092-1101.

[77] H. Yan, Z.H. Chen, Y. Zheng, C. Newman, J.R. Quinn, F. Dotz, M. Kastler, A. Facchetti, Nature, 457 (2009) 679-686.

[78] J. Rivnay, R. Steyrleuthner, L.H. Jimison, A. Casadei, Z.H. Chen, M.F. Toney, A. Facchetti, D. Neher, A. Salleo, Macromolecules, 44 (2011) 5246-5255.

[79] M. Caironi, M. Bird, D. Fazzi, Z. Chen, R. Di Pietro, C. Newman, A. Facchetti, H. Sirringhaus, Advanced Functional Materials, 21 (2011) 3371-3381.

[80] R. Steyrleuthner, R. Di Pietro, B.A. Collins, F. Polzer, S. Himmelberger, M. Schubert, Z. Chen, S. Zhang, A. Salleo, H. Ade, A. Facchetti, D. Neher, J Am Chem Soc, 136 (2014) 42454256.

[81] Y.M. Gross, D. Trefz, R. Tkachov, V. Untilova, M. Brinkmann, G.L. Schulz, S. Ludwigs, Macromolecules, 50 (2017) 5353-5366.

[82] D. Trefz, A. Ruff, R. Tkachoy, M. Wieland, M. Goll, A. Kiriy, S. Ludwigs, Journal of Physical Chemistry C, 119 (2015) 22760-22771.

[83] S.H. Wang, H.D. Sun, U. Ail, M. Vagin, P.O.A. Persson, J.W. Andreasen, W. Thiel, M. Berggren, X. Crispin, D. Fazzi, S. Fabiano, Advanced Materials, 28 (2016) 10764-10771.

[84] R. Matsidik, A. Luzio, O. Askin, D. Fazzi, A. Sepe, U. Steiner, H. Komber, M. Caironi, M. Sommer, Chem Mater, 29 (2017) 5473-5483.

[85] S.H. Wang, H.D. Sun, T. Erdmann, G. Wang, D. Fazzi, U. Lappan, Y. Puttisong, Z.H. Chen, M. Berggren, X. Crispin, A. Kiriy, B. Voit, T.J. Marks, S. Fabiano, A. Facchetti, Advanced Materials, 30 (2018) 1801898.

[86] R. Steyrleuthner, M. Schubert, I. Howard, B. Klaumunzer, K. Schilling, Z. Chen, P. Saalfrank, F. Laquai, A. Facchetti, D. Neher, J Am Chem Soc, 134 (2012) 18303-18317.

[87] S.H. Wang, S. Fabiano, S. Himmelberger, S. Puzinas, X. Crispin, A. Salleo, M. Berggren, Proceedings of the National Academy of Sciences of the United States of America, 112 (2015) 10599-10604. 
[88] L. Burgi, M. Turbiez, R. Pfeiffer, F. Bienewald, H.J. Kirner, C. Winnewisser, Advanced Materials, 20 (2008) 2217-2224.

[89] X. Zhang, L.J. Richter, D.M. DeLongchamp, R.J. Kline, M.R. Hammond, I. McCulloch, M. Heeney, R.S. Ashraf, J.N. Smith, T.D. Anthopoulos, B. Schroeder, Y.H. Geerts, D.A. Fischer, M.F. Toney, J Am Chem Soc, 133 (2011) 15073-15084.

[90] Z.Y. Chen, M.J. Lee, R.S. Ashraf, Y. Gu, S. Albert-Seifried, M.M. Nielsen, B. Schroeder, T.D. Anthopoulos, M. Heeney, I. McCulloch, H. Sirringhaus, Advanced Materials, 24 (2012) 647-652.

[91] H.J. Chen, Y.L. Guo, G. Yu, Y. Zhao, J. Zhang, D. Gao, H.T. Liu, Y.Q. Liu, Advanced Materials, 24 (2012) 4618-4622.

[92] J.D. Yuen, J. Fan, J. Seifter, B. Lim, R. Hufschmid, A.J. Heeger, F. Wudl, Journal of the American Chemical Society, 133 (2011) 20799-20807.

[93] A.J. Kronemeijer, E. Gili, M. Shahid, J. Rivnay, A. Salleo, M. Heeney, H. Sirringhaus, Advanced Materials, 24 (2012) 1558-1565.

[94] M. Shahid, T. McCarthy-Ward, J. Labram, S. Rossbauer, E.B. Domingo, S.E. Watkins, N. Stingelin, T.D. Anthopoulos, M. Heeney, Chemical Science, 3 (2012) 181-185.

[95] R. Stalder, J.G. Mei, J.R. Reynolds, Macromolecules, 43 (2010) 8348-8352.

[96] E.G. Wang, Z.F. Ma, Z. Zhang, K. Vandewal, P. Henriksson, O. Inganas, F.L. Zhang, M.R. Andersson, Journal of the American Chemical Society, 133 (2011) 14244-14247.

[97] T. Lei, Y. Cao, Y.L. Fan, C.J. Liu, S.C. Yuan, J. Pei, Journal of the American Chemical Society, 133 (2011) 6099-6101.

[98] T. Lei, J.H. Dou, J. Pei, Advanced Materials, 24 (2012) 6457-6461.

[99] J.G. Mei, Z.N. Bao, Chemistry of Materials, 26 (2014) 604-615.

[100] T. Lei, J.H. Dou, Z.J. Ma, C.H. Yao, C.J. Liu, J.Y. Wang, J. Pei, Journal of the American Chemical Society, 134 (2012) 20025-20028.

[101] R.P. Fornari, A. Troisi, Adv Mater, 26 (2014) 7627-7631.

[102] S.G. Bucella, A. Luzio, E. Gann, L. Thomsen, C.R. McNeill, G. Pace, A. Perinot, Z.H. Chen, A. Facchetti, M. Caironi, Nature Communications, 6 (2015) 8394.

[103] X.D. Gu, L. Shaw, K. Gu, M.F. Toney, Z.N. Bao, Nature Communications, 9 (2018) 534. [104] H.Y. Wang, Y.Z. Xu, X.H. Yu, R.B. Xing, J.G. Liu, Y.C. Han, Polymers, 5 (2013) 12721324.

[105] D.M. DeLongchamp, R.J. Kline, E.K. Lin, D.A. Fischer, L.J. Richter, L.A. Lucas, M. Heeney, I. McCulloch, J.E. Northrup, Advanced Materials, 19 (2007) 833-837.

[106] S.A. Mollinger, B.A. Krajina, R. Noriega, A. Salleo, A.J. Spakowitz, ACS Macro Letters, 4 (2015) 708-712.

[107] D.T. Duong, V. Ho, Z.R. Shang, S. Mollinger, S.C.B. Mannsfeld, J. Dacuna, M.F. Toney, R. Segalman, A. Salleo, Advanced Functional Materials, 24 (2014) 4515-4521.

[108] P. Pingel, A. Zen, R.D. Abellon, F.C. Grozema, L.D.A. Siebbeles, D. Neher, Advanced Functional Materials, 20 (2010) 2286-2295.

[109] J.C. Bolsee, W.D. Oosterbaan, L. Lutsen, D. Vanderzande, J. Manca, Advanced Functional Materials, 23 (2013) 862-869.

[110] A.M. Hindeleh, R. Hosemann, Journal of Materials Science, 26 (1991) 5127-5133.

[111] A.M. Hindeleh, R. Hosemann, Journal of Physics C-Solid State Physics, 21 (1988) 4155 4170.

[112] J. Rivnay, R. Noriega, R.J. Kline, A. Salleo, M.F. Toney, Physical Review B, 84 (2011) 121306.

[113] R.A. Street, J.E. Northrup, A. Salleo, Physical Review B, 71 (2005) 165202.

[114] S. Himmelberger, A. Salleo, MRS Communications, 5 (2015) 383-395.

[115] T. Marszalek, M.M. Li, W. Pisula, Chemical Communications, 52 (2016) 10938-10947. 
[116] B. McCulloch, V. Ho, M. Hoarfrost, C. Stanley, C. Do, W.T. Heller, R.A. Segalman, Macromolecules, 46 (2013) 1899-1907.

[117] W.L. Zhang, E.D. Gomez, S.T. Milner, Macromolecules, 47 (2014) 6453-6461.

[118] A.C. Grimsdale, K. Müllen, Macromolecular Rapid Communications, 28 (2007) 16761702.

[119] L.H. Zhao, R.Q. Png, J.M. Zhuo, L.Y. Wong, J.C. Tang, Y.S. Su, L.L. Chua, Macromolecules, 44 (2011) 9692-9702.

[120] B. Kuei, E.D. Gomez, Soft Matter, 13 (2017) 49-67.

[121] P. Carbone, A. Troisi, Journal of Physical Chemistry Letters, 5 (2014) 2637-2641.

[122] W.C. Germs, K. Guo, R.A.J. Janssen, M. Kemerink, Physical Review Letters, 109 (2012) 056601 .

[123] D. Venkateshvaran, A.J. Kronemeijer, J. Moriarty, D. Emin, H. Sirringhaus, Apl Materials, 2 (2014) 032102.

[124] X.L. Li, X.R. Wang, L. Zhang, S.W. Lee, H.J. Dai, Science, 319 (2008) 1229-1232.

[125] L. Chen, Y. Hernandez, X.L. Feng, K. Müllen, Angewandte Chemie-International Edition, 51 (2012) 7640-7654.

[126] C.J. Takacs, N.D. Treat, S. Kramer, Z. Chen, A. Facchetti, M.L. Chabinyc, A.J. Heeger, Nano Lett, 13 (2013) 2522-2527.

[127] S. Goffri, C. Muller, N. Stingelin-Stutzmann, D.W. Breiby, C.P. Radano, J.W. Andreasen, R. Thompson, R.A.J. Janssen, M.M. Nielsen, P. Smith, H. Sirringhaus, Nature Materials, 5 (2006) 950-956.

[128] L.Z. Qiu, W.H. Lee, X.H. Wang, J.S. Kim, J.A. Lim, D. Kwak, S. Lee, K. Cho, Advanced Materials, 21 (2009) 1349-1353.

[129] G. Lu, J. Blakesley, S. Himmelberger, P. Pingel, J. Frisch, I. Lieberwirth, I. Salzmann, M. Oehzelt, R. Di Pietro, A. Salleo, N. Koch, D. Neher, Nat Commun, 4 (2013) 1588.

[130] S.H. Wang, W. Pisula, K. Müllen, J Mater Chem, 22 (2012) 24827-24831.

[131] S. Han, X. Yu, W. Shi, X. Zhuang, J. Yu, Organic Electronics, 27 (2015) 160-166.

[132] D. Chlebosz, Ł. Janasz, K. Janus, M. Gazińska, W. Goldeman, J. Ulanski, W. Pisula, A. Kiersnowski, Dyes and Pigments, 140 (2017) 491-499.

[133] G. Wang, W. Huang, N.D. Eastham, S. Fabiano, E.F. Manley, L. Zeng, B. Wang, X. Zhang, Z. Chen, R. Li, R.P.H. Chang, L.X. Chen, M.J. Bedzyk, F.S. Melkonyan, A. Facchetti, T.J. Marks, Proc Natl Acad Sci U S A, 114 (2017) E10066-E10073.

[134] J. Xu, S.H. Wang, G.J.N. Wang, C.X. Zhu, S.C. Luo, L.H. Jin, X.D. Gu, S.C. Chen, V.R. Feig, J.W.F. To, S. Rondeau-Gagne, J. Park, B.C. Schroeder, C. Lu, J.Y. Oh, Y.M. Wang, Y.H. Kim, H. Yan, R. Sinclair, D.S. Zhou, G. Xue, B. Murmann, C. Linder, W. Cai, J.B.H. Tok, J.W. Chung, Z.N. Bao, Science, 355 (2017) 59-64.

[135] G.Y. Zhang, M. McBride, N. Persson, S. Lee, T.J. Dunn, M.F. Toney, Z.B. Yuan, Y.H. Kwon, P.H. Chu, B. Risteen, E. Reichmanis, Chemistry of Materials, 29 (2017) 7645-7652.

[136] G. Zhang, S. Lee, E. Gutiérrez-Meza, C. Buckley, M. McBride, D.A. Valverde-Chávez, Y.H. Kwon, V. Savikhin, H. Xiong, T.J. Dunn, M.F. Toney, Z. Yuan, C. Silva, E. Reichmanis, Chemistry of Materials, (2019) 10.1021/acs.chemmater.8b05224.

[137] S.Y. Son, Y. Kim, J. Lee, G.Y. Lee, W.T. Park, Y.Y. Noh, C.E. Park, T. Park, J Am Chem Soc, 138 (2016) 8096-8103.

[138] K.H. Park, S.Y. Son, J.O. Kim, G. Kang, T. Park, D. Kim, Journal of Physical Chemistry Letters, 9 (2018) 3173-3180.

[139] S.Y. Son, J.H. Kim, E. Song, K. Choi, J. Lee, K. Cho, T.S. Kim, T. Park, Macromolecules, 51 (2018) 2572-2579.

[140] S.E. Root, S. Savagatrup, A.D. Printz, D. Rodriquez, D.J. Lipomi, Chemical Reviews, 117 (2017) 6467-6499. 
[141] S.H. Wang, J.Y. Oh, J. Xu, H. Tran, Z.N. Bao, Accounts of Chemical Research, 51 (2018) 1033-1045.

[142] H. Sirringhaus, Advanced Materials, 21 (2009) 3859-3873.

[143] P.A. Bobbert, A. Sharma, S.G.J. Mathijssen, M. Kemerink, D.M. de Leeuw, Advanced Materials, 24 (2012) 1146-1158.

[144] M. Nikolka, I. Nasrallah, B. Rose, M.K. Ravva, K. Broch, A. Sadhanala, D. Harkin, J. Charmet, M. Hurhangee, A. Brown, S. Illig, P. Too, J. Jongman, I. McCulloch, J.L. Bredas, H. Sirringhaus, Nature Materials, 16 (2017) 356-362.

[145] A. Onwubiko, W. Yue, C. Jellett, M.F. Xiao, H.Y. Chen, M.K. Ravva, D.A. Hanifi, A.C. Knall, B. Purushothaman, M. Nikolka, J.C. Flores, A. Salleo, J.L. Bredas, H. Sirringhaus, P. Hayoz, I. McCulloch, Nature Communications, 9 (2018) 416. 


\section{Graphical abstract}

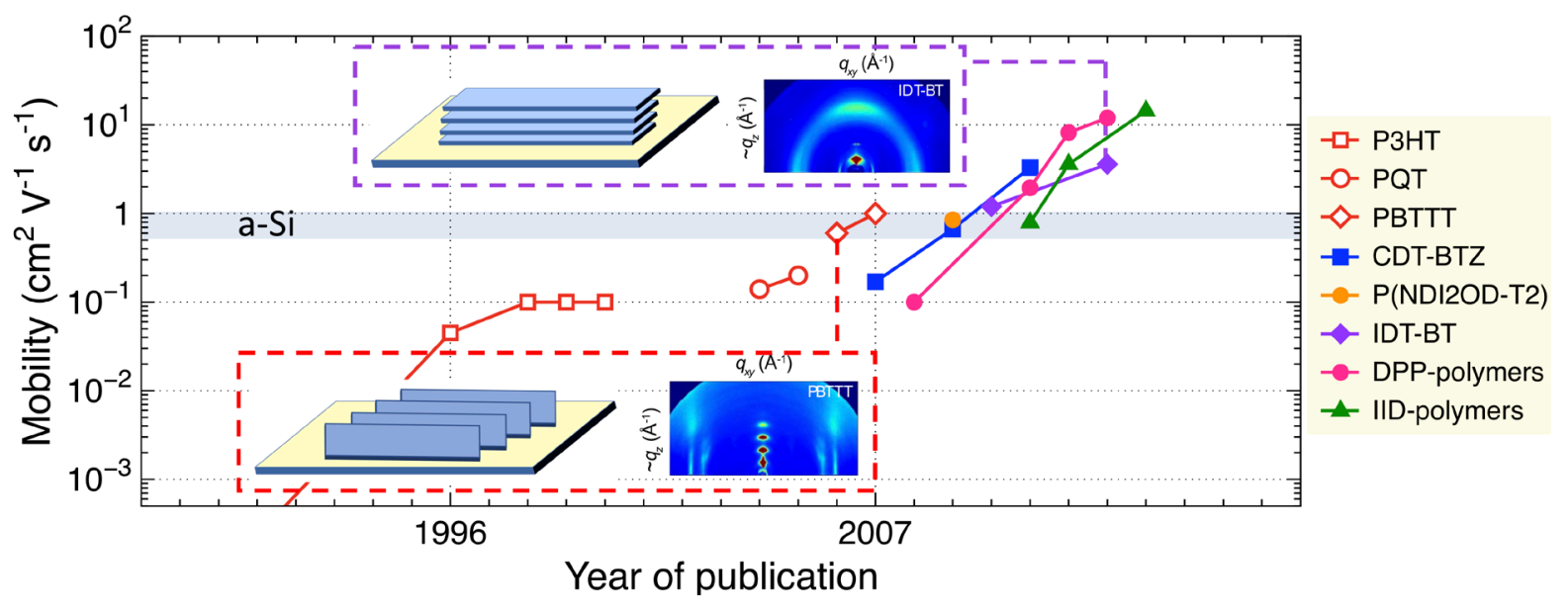

